\title{
PHOTOIONIZATION FROM THE Xe 4d ORBITALS OF $\mathrm{XeF}_{2}$
}

\author{
R. Forbes, ${ }^{a}$ P. Hockett, ${ }^{b}$ I. Powis, ${ }^{c}$ J. D. Bozek, ${ }^{\text {d }}$ D. M. P. Holland, ${ }^{e}$ and S. T. Pratt ${ }^{f *}$
}

aPULSE Institute, SLAC National Accelerator Laboratory, 2575 Sand Hill Road, Menlo Park,

$$
\text { CA } 94025 \text { USA }
$$

${ }^{\mathrm{b}}$ National Research Council of Canada, 100 Sussex Dr, Ottawa, ON K1A 0R6, Canada

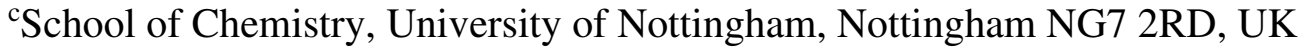

${ }^{\mathrm{d} S y n c h r o t r o n ~ S O L E I L, ~ l ' O r m e ~ d e s ~ M e r i s i e r s, ~ S a i n t-A u b i n, ~ B P ~ 48, ~} 91192$ Gif-sur-Yvette, France

e'Daresbury Laboratory, Daresbury, Warrington, Cheshire, WA4 4AD, UK

${ }_{\mathrm{f}}^{\mathrm{f}}$ Chemical Sciences and Engineering Division, Argonne National Laboratory, Lemont, IL 60439 USA

*Corresponding Author Email: stpratt@anl.gov

\begin{abstract}
The submitted manuscript has been created by UChicago Argonne, LLC, Operator of Argonne National Laboratory ("Argonne"). Argonne, a U.S. Department of Energy Office of Science laboratory, is operated under Contract No. DEAC02-06CH11357. The U.S. Government retains for itself, and others acting on its behalf, a paid-up nonexclusive, irrevocable worldwide license in said article to reproduce, prepare derivative works, distribute copies to the public, and perform publicly and display publicly, by or on behalf of the Government.
\end{abstract}




\begin{abstract}
We present a comparison of the photoionization dynamics of the $4 \mathrm{~d}$ shell of $\mathrm{XeF}_{2}$ from threshold to $250 \mathrm{eV}$ to those of the prototypical $4 \mathrm{~d}$ shell of atomic Xe. The new experimental data include spin-orbit and ligand-field resolved partial cross sections, photoelectron angular distributions, branching fractions, and lifetime widths for the $4 \mathrm{~d}$-hole states. The spin-orbit branching fractions and angular distributions are remarkably similar to the corresponding distributions from atomic Xe across a broad energy interval that includes both the intense shape resonance in the $\mathrm{f}$ continuum and a Cooper minimum in the same channel. The angular distributions and branching fractions are also in reasonably good agreement with our first-principles theoretical calculations on $\mathrm{XeF}_{2}$. Data are also presented on the lifetime widths of the substate-resolved 4d-hole states of $\mathrm{XeF}_{2}$. While the trends in the widths are similar to those in earlier experimental and theoretical work, the linewidths are considerably smaller than in the previous measurements, which may require some reinterpretation of the decay mechanism. Finally, we present new data and an analysis of the Auger electron spectra for ionization above the $4 \mathrm{~d}$ thresholds, as well as resonant Auger spectra for several pre-edge features.
\end{abstract}




\section{INTRODUCTION}

Photoexcitation and ionization from the $4 \mathrm{~d}$ shell of atomic Xe has long been a touchstone for experimental and theoretical studies of shape resonances, Cooper minima, and interchannel coupling. ${ }^{1,2,3,4,5,6,7,8,9,10,11,12,13,14,15,16,17,18,19,20,21,22,23}$ Removal of a 4d electron from Xe results in two spin-orbit states, ${ }^{2} \mathrm{D}_{5 / 2}$ and ${ }^{2} \mathrm{D}_{3 / 2}$, with thresholds of 67.548(11) $\mathrm{eV}$ and 69.537(12) eV, respectively. ${ }^{5}$ Experiment and theory have both addressed the corresponding photoabsorption and photoionization cross sections,,${ }^{1,2,3,4,5,6,13,20}$ photoelectron angular distributions, ${ }^{9,10}$ and spinorbit branching ratios. ${ }^{4,18}$ The $4 \mathrm{~d}$ hole states lie above the double- and triple-ionization thresholds of $\mathrm{Xe}\left(33.105 \pm 0.004 \mathrm{eV}\right.$ and $64.09 \pm 0.04 \mathrm{eV}$, respectively $\left.{ }^{24,25}\right)$, and the decay processes of the 4d-hole states have also been the subject of intense study, with determinations of Auger decay widths, ${ }^{21,22}$ final-state branching ratios, and Auger electron angular distributions. ${ }^{15,26}$ Photoelectron-Auger electron coincidence and Auger-electron ion coincidence measurements have been particularly effective for characterizing the multiple decay pathways. ${ }^{27,28,29}$

The evolution of structure and dynamics from those of a free atom to those of a molecule can provide deep insight into the nature of chemical bonding, particularly when they are studied as a function of the molecular orbital - from outer-valence, to inner-valence, to outer-core and innercore orbitals. One of the most interesting questions about the outer-core electrons is the extent to which they are affected by the molecular environment and participate in molecular bonding. From this perspective, the xenon fluorides, $\mathrm{XeF}_{n}(n=2,4,6)$ represent a particularly intriguing set of relatively weakly bound molecules in which the perturbation of the Xe inner-shell electrons by the highly electrophilic fluorine ligands ${ }^{30,31,32}$ can be characterized with considerable detail and benchmarked against the wealth of data on atomic Xe.

In this paper, we focus on the photoexcitation of the $4 \mathrm{~d}$ subshell of $\mathrm{XeF}_{2}$ between $60 \mathrm{eV}$ and 250 $\mathrm{eV}$, and on the decay of the $4 \mathrm{~d}^{-1}$ hole states. The present work builds on a long history of work on the xenon fluorides. Comes et al. ${ }^{31}$ recorded the first extreme ultraviolet (XUV) 
photoabsorption spectrum of $\mathrm{XeF}_{2}$ and $\mathrm{XeF}_{4}$ between 50 and $160 \mathrm{eV}$, a region dominated by bound resonances just below the $\mathrm{Xe} 4 \mathrm{~d}^{-1}$ threshold and by a large $4 \mathrm{~d} \rightarrow \varepsilon \mathrm{f}$ shape resonance in the region above it. This work was followed by photoelectron spectroscopy studies of $\mathrm{XeF}_{2}, \mathrm{XeF}_{4}$, and $\mathrm{XeF}_{6}$ by Carroll et al. ${ }^{32}$ and Bancroft et al. ${ }^{33}$ at the $\mathrm{F} 1 \mathrm{~s}$ and $\mathrm{Xe} 3 \mathrm{~d}$ thresholds. Auger electron spectroscopy following Xe 3d hole formation was also characterized by Aksela et al., ${ }^{34}$ and most recently Southworth et al. ${ }^{35}$ performed a detailed study of the fragmentation dynamics of $\mathrm{XeF}_{2}$ following $3 \mathrm{~d}$ hole production. At lower energy, Cutler et al. ${ }^{36}$ have used high-resolution photoelectron spectroscopy to characterize the ligand-field and spin-orbit splittings of the Xe $4 \mathrm{~d}$ hole states in $\mathrm{XeF}_{2}, \mathrm{XeF}_{4}$, and $\mathrm{XeF}_{6}$. Cutler et al. ${ }^{36}$ could explain the observed ligand-field splittings with an instructive model for the bonding, and they also found different lifetime widths for the different ligand-field-split substates. Bancroft et al ${ }^{37}$ later studied the interchannel coupling among the valence continua and the $4 \mathrm{~d}$ excitation channel between 50 and $110 \mathrm{eV}$, and found good agreement with their theoretical predictions.

The earliest theoretical work on $\mathrm{XeF}_{2}$ was performed by Basch et al., ${ }^{30}$ and was aimed at the characterization of the ordering of the molecular orbitals and the nature of the bonding. The currently accepted ordering of the configuration of $\mathrm{XeF}_{2}$ is: ...(Xe $\left.4 s\right)^{2}(\mathrm{Xe} 4 \mathrm{p})^{6}(\mathrm{Xe}$ $4 \mathrm{~d})^{10} 8 \sigma_{\mathrm{g}}{ }^{2} 5 \sigma_{\mathrm{u}}{ }^{2} 9 \sigma_{\mathrm{g}}{ }^{2} 6 \sigma_{\mathrm{u}}{ }^{2} 4 \pi_{\mathrm{u}}{ }^{4} 3 \pi_{\mathrm{g}}{ }^{4} 10 \sigma_{\mathrm{g}}{ }^{2} 5 \pi_{\mathrm{u}}{ }^{4}{ }^{33}$ More recently, Buth et al. ${ }^{38}$ performed detailed calculations on the single-ionization spectra of $\mathrm{XeF}_{2}, \mathrm{XeF}_{4}$, and $\mathrm{XeF}_{6}$ from the first ionization energy to the Xe $4 \mathrm{~d}$ ionization threshold. In a subsequent paper, ${ }^{39}$ they performed calculations and analysis of the double ionization thresholds, as well as a study of the decay mechanisms for the Xe $4 \mathrm{~d}$ hole states. One of the interesting observations of Cutler et al. ${ }^{36}$ was that the Auger decay widths, $\Gamma$, of the $\mathrm{Xe} 4 \mathrm{~d}$ hole states increased with the addition of fluorine with $\mathrm{Xe}<\mathrm{XeF}_{2}$ $<\mathrm{XeF}_{4}<\mathrm{XeF}_{6}$. Buth et al. explained this trend in terms of an increasingly important contribution from interatomic Coulombic decay to the Auger process. In later work, Pernpointner and Cederbaum $^{40}$ assessed the role of relativistic effects on the ionization spectra of these molecules. 
A second theoretical study of $\mathrm{XeF}_{2}$ including relativistic effects has been reported by Haiduke et al. $^{41}$

Here, we present new ion-yield and high-resolution photoelectron spectra that result in a comprehensive dataset for the $4 \mathrm{~d}^{-1}$ partial photoionization cross sections for $\mathrm{XeF}_{2}$, as well as the photon-energy dependence of the $\mathrm{XeF}_{2} 4 \mathrm{~d}^{-1}$ substate branching fractions and photoelectron angular distributions. These data are compared with the earlier results, as well as with new theoretical calculations, and with the vast data on atomic Xe. The photoelectron spectra also allow a more detailed examination of the substate-specific widths and Auger decay lifetimes for the $\mathrm{XeF}_{2} 4 \mathrm{~d}^{-1}$ hole, as well as a more detailed comparison with theory. Auger-electron and resonant Auger-electron spectra are also reported that provide additional insight into the decay mechanisms of the $4 \mathrm{~d}^{-1}$ hole. This study complements our recent comparisons of the $3 \mathrm{~d}$ and $4 \mathrm{~d}$ photoexcitation of $\mathrm{Xe}$ and methyl iodide, $\mathrm{CH}_{3} \mathrm{I}$, a more strongly bound system that is isoelectronic with $\mathrm{Xe}^{42,43}$

In what follows, the experimental and theoretical methodologies are described first, followed by a presentation of the experimental data. The discussion begins with the ion-yield spectra and partial cross sections, and continues on to the $4 \mathrm{~d}^{-1}$ ligand-field and spin-orbit dependent branching fractions and photoelectron angular distributions. The $4 \mathrm{~d}^{-1}$ substate-dependent Augerlifetime widths are then discussed. The Auger electron spectra and resonant Auger electron spectra are then discussed using insights from the calculations of Buth et al. ${ }^{38,39}$ Finally, the present findings are discussed in the context of the growing number of pump-probe experiments performed by using new ultrafast sources of XUV and x-ray radiation.

\section{EXPERIMENT}

The experiments were performed on the PLEIADES beamline at the Synchrotron SOLEIL. The experimental approach was similar to that of our previous studies of the I $4 \mathrm{~d}$ subshell of $\mathrm{CH}_{3} \mathrm{I},{ }^{43}$ 
and is described only briefly here. The linearly polarized radiation from the Apple II undulator was passed through a modified Petersen monochromator and into the interaction region of a VG Scienta R4000 electron spectrometer. The undulator radiation was determined to be $>99 \%$ plane polarized, and the plane was rotated to be either parallel or perpendicular to the entrance axis of the electron spectrometer. We refer to the electron signal in these two geometries as $I_{0}$ and $I_{90}$, respectively. The incident monochromatic photon intensity was measured with a photodiode mounted downstream of the electron spectrometer.

The major change to the experimental setup compared to that employed previously ${ }^{44}$ was the replacement of the standard Scienta ionization cell with one, designed in-house, allowing a significantly reduced pumping capacity, and hence sample throughput. A schematic diagram of the interaction region is included in the Supplemental Material. The ion yield spectra were measured in a separate chamber where the sample was introduced into the interaction region as an effusive jet through an needle positioned $\sim 1 \mathrm{~mm}$ above the photon beam. A channeltron with a $2 \mathrm{~cm}$ opening was positioned $\sim 1 \mathrm{~cm}$ from the intersection of the two beams in the horizontal plane. The front of the channeltron was biased with $-2000 \mathrm{~V}$, effectively extracting all positive photoions created in the interaction region. The ion signal from the channeltron was amplified, discriminated and counted for a set dwell time of 1 s per point. The inlet system for the $\mathrm{XeF}_{2}$ sample was modified to minimize the distance between the sample holder and the ionization cell. The inlet line was extensively passivated to minimize the contribution of atomic Xe to the electron signal. The residual lines from atomic Xe could be used to calibrate both the photon energy and the electron kinetic energy. In addition, a series of photoelectron spectra was recorded using a mixed sample with roughly equal pressures of $\mathrm{XeF}_{2}$ and $\mathrm{Xe}$. While the emphasis of our measurements was on electron spectroscopy, total ion yield spectra were also recorded for $\mathrm{XeF}_{2}$. These spectra were used to choose the photon energies for the resonant Auger studies. 
Photoelectron spectra of $\mathrm{XeF}_{2}$ were recorded at photon energies between $\sim 60$ and $250 \mathrm{eV}$, resulting in electrons with energies up to $240 \mathrm{eV}$. Most of the measurements focused on the $4 \mathrm{~d}^{-1}$ photolines, although spectra were also recorded at selected energies to observe electrons produced by shake-up processes, Auger decay, resonant Auger processes, and valence-shell photoionization. All of the spectra have been corrected for the transmission function of the electron spectrometer. The monochromator and electron spectrometer resolutions were optimized for the different measurements depending on the signal levels and the achievable signal-to-noise level.

In the electric dipole approximation with plane-polarized light, the differential partial cross section is given by: ${ }^{45}$

$$
\frac{d}{d}=\frac{-}{4}\left[1+P_{2}(\cos )\right]
$$

where $\sigma$ is the angle-integrated partial cross section, $\beta$ is the angular distribution parameter that takes values between -1 and $2, P_{2}(\cos \theta)$ is the second-order Legendre polynomial, and $\theta$ is the angle between the plane of polarization and the plane of the ejected electron momentum. Using Equation 1 to express $\beta$ in terms of the measurements at $0^{\circ}$ and $90^{\circ}$, one finds:

$$
=\frac{2\left(\begin{array}{ll}
I_{0} & I_{90}
\end{array}\right)}{\left(\begin{array}{l}
I_{0}+2 I_{90}
\end{array}\right)},
$$

which is used in the present determinations. The entrance slit of the analyzer limits the acceptance angle in the dispersive direction, which is the relevant angle for $\beta$ measurements. Taking into account the geometry of the instrument, the size and curvature of the slits, and the magnifying effect of the spectrometer lens, the angular acceptance in the measurement is approximately $0.5^{\circ}$, which results in a contribution to the uncertainty of $\sim 1-2 \%$. 


\section{B. Fitting}

The photoelectron spectra of the $\mathrm{XeF}_{2} 4 \mathrm{~d}^{-1}$ photolines were fitted to extract the substate binding energies, peak intensities and widths, and $\beta$ parameters. As described in detail previously, ${ }^{42,43,44} \mathrm{a}$ weighted nonlinear least-squares procedure was used to fit the five components (three for $4 d_{5 / 2}{ }^{-1}$ and two for $4 \mathrm{~d}_{3 / 2^{-1}}$ ) for the $4 \mathrm{~d}^{-1}$ hole. In the case of the $\mathrm{Xe}+\mathrm{XeF}_{2}$ mixtures, the two $\mathrm{Xe} 4 \mathrm{~d}^{-1}$ lines and the five $\mathrm{XeF}_{2}$ were all fit simultaneously. The error bars derived from the fits represent only the counting statistics, and do not include systematic errors. As discussed by Cutler et al., ${ }^{36}$ vibrational excitation in the $4 \mathrm{~d}^{-1}$ hole spectra is expected to be a minor process, and no vibrational structure was considered. The procedure involved the simultaneous fitting of the photoelectron spectra obtained with both parallel and perpendicular polarizations with respect to the entrance slit of the electron spectrometer. In addition, at each photon energy, an experimental broadening parameter was used for all five (or seven) transitions that took into account the electron spectrometer resolution, photon bandwidth, and Doppler broadening. In contrast with our previous studies, ${ }^{42,43,44}$ all five photoelectron peak widths were allowed to vary separately. The output of the fits thus provided the ligand-field- and spin-orbit-split substate-specific angular distribution parameters, peak intensities, and linewidths that form the basis for the discussion of the $4 \mathrm{~d}^{-1}$ photoionization processes and decay mechanisms.

\section{Computational Methods}

The electronic structure was computed, at the HF/SPKrQZP level, in Gamess (version 2018 R3), ${ }^{46,47}$ and converged to an equilibrium bond-length of $1.9373 \AA$, slightly shorter than the experimental gas-phase value of 1.977(15) $\AA$ obtained via high-resolution spectroscopy. ${ }^{48}$ Scattering calculations made use of the ePolyScat suite, ${ }^{49,50,51}$ which computes the fixed-nuclei scattering wavefunction via a Schwinger variational procedure. Although the calculations were performed in $\mathrm{D}_{8 \mathrm{~h}}$ symmetry, here we use the corresponding $\mathrm{D}_{\infty \mathrm{h}}$ labels appropriate for $\mathrm{XeF}_{2}$. Orbitals 21-25 correspond to the $\mathrm{Xe}(4 \mathrm{~d})$ core levels, and comprise a single $\Sigma_{\mathrm{g}}$ orbital (oriented along the bond), and two doubly degenerate orbitals $\Pi_{\mathrm{g}}$ and $\Delta_{\mathrm{g}}$. The orbital energies were 
computed to be $\Sigma_{\mathrm{g}}=-76.58, \Pi_{\mathrm{g}}=-76.27$ and $\Delta_{\mathrm{g}}=-75.67 \mathrm{eV}$ respectively, which is approximately $6 \mathrm{eV}$ from our experimentally measured ligand-field-averaged energy for the lower spin-orbit component of $\sim 70.38 \mathrm{eV}$ ( $\sim 72.39 \mathrm{eV}$ for the upper spin-orbit component). To allow for the bond-length dependence of the scattering (see, for example, References 52, 53, 54, and 55) and to (crudely) approximate symmetric stretching vibrational effects, the results were computed for a range of Xe-F bond lengths about the equilibrium value, and averaged over these via a Gaussian convolution kernel with $\mathrm{s}=0.2 \AA$. No treatment for the symmetry-breaking bend and asymmetric stretching was applied, although due to its low frequency the former is likely to be thermally excited in our room-temperature measurements. Full computational results and additional notes and analysis are available online via Reference 56.

It is of note that the present calculations do not include multi-electron effects such as the Xe $4 \mathrm{~d}$ $\rightarrow$ ff giant resonance. ${ }^{57}$ As a result, the broad width of the resonances near $100 \mathrm{eV}$ in $\mathrm{Xe}$ and $\mathrm{XeF}_{2}$ is not reproduced in the ePolyScat results. An illuminating comparison can be found in Reference 58, where multi-electron effects are investigated using a relativistic random-phase approximation (RRPA) treatment, and good agreement with the observed absolute cross sections is obtained for a range of isoelectronic atoms. Previous studies on atomic Xe have also shown that calculations including final-state correlation peaked at an energy somewhat above the experiment, but reproduced the width of the resonance reasonably well. ${ }^{2,56}$

Spin-orbit effects were added to the computational results by introducing an additional spin-orbit coupling term that describes the final ionic states, as has been demonstrated in previous work. ${ }^{59,60}$ This approach makes use of an angular momentum coupling term, $\mathrm{C}^{\mathrm{SO}}$, defined as follows:

$$
C^{\mathrm{SO}}(L, J, S)=\left(\begin{array}{ccc}
L & J & S \\
M_{L} & M_{J} & M_{S}
\end{array}\right)\left(\begin{array}{lll}
L & J & S
\end{array}\right),
$$


where the notation follows Hund's case b/c, with the lab-frame (LF) projections denoted $M_{L, J, S}$ and the molecular frame (MF) projections denoted $\Lambda, \Omega$, and $\Sigma$. The observables then include a term defined by the coherent product over coupling terms, summed over any unobserved states, v:

$$
(L, J, S)=C^{\mathrm{SO}}(L, J, S) C^{\mathrm{SO}}\left(L^{\prime}, J^{\prime}, S^{\prime}\right)
$$

In the current case, this term has been applied to the ePolyScat computed matrix elements to generate the spin-orbit split bands with the use of several assumptions. First, all lab-frame $M$ sub-levels are assumed to be equally populated, and thus the lab-frame term will not affect the relative spin-orbit states. Second, the ion states are assumed to be fully resolved, so that there are no cross-terms (coherences). The quantum numbers for the $4 \mathrm{~d}^{-1}$ hole state take the values $\mathrm{L}=2$, $S=1 / 2$, and $J=L+S$, resulting in the ${ }^{2} \mathrm{D}_{5 / 2}$ and ${ }^{2} \mathrm{D}_{3 / 2}$ terms. The ligand-field split components have $\Lambda=0-2$, corresponding to the $\Sigma, \Pi$, and $\Delta$ components, respectively, which correspond to the $\Sigma_{\mathrm{g}}, \Pi_{\mathrm{g}}$, and $\Delta_{\mathrm{g}}$ states of the calculations. $\Omega$ corresponds to the allowed projection terms, subselected to match the experimentally assigned values. Finally, no spin-orbit energy splitting is computed, and terms are assumed to be energetically ordered as in the experimental assignments. With this approach, appropriate sub-selection and multiplication of the computational cross sections, followed by renormalization, yields the effective spin-orbit branching ratios in the oneelectron model.

\section{RESULTS AND DISCUSSION}

Figure 1 shows the photoelectron spectrum of the $4 \mathrm{~d}^{-1}$ region for a mixture of $\mathrm{Xe}$ and $\mathrm{XeF}_{2}$ recorded at a photon energy of $115 \mathrm{eV}$ and with $\theta=0^{\circ}$, along with the results of the fitting procedure. As discussed by Cutler et al., ${ }^{36}$ the $\mathrm{XeF}_{2} 4 \mathrm{~d}^{-1}$ threshold shifts to approximately $2 \mathrm{eV}$ higher energy than the corresponding atomic $\mathrm{Xe}_{4} \mathrm{~d}^{-1}$ thresholds. As in $\mathrm{Xe}$, the $\mathrm{XeF}_{2} 4 \mathrm{~d}^{-1}$ threshold is split into two spin-orbit components, corresponding to the $4 \mathrm{~d}_{5} / 2^{-1}$ and $4 \mathrm{~d}_{3} / 2^{-1}$ levels. Furthermore, each of these levels is split by ligand-field interactions. Using the results from our 
fitting procedure, the lower $4 \mathrm{~d}_{5 / 2}{ }^{-1}$ threshold is split into ${ }^{2} \Delta_{5 / 2},{ }^{2} \Pi_{3 / 2}$, and ${ }^{2} \Sigma_{1 / 2}$ states with energies of $70.169 \mathrm{eV}, 70.404 \mathrm{eV}$, and $70.579 \mathrm{eV}$, respectively, while the higher energy $4 \mathrm{~d}_{3 / 2}{ }^{-1}$ threshold is split into ${ }^{2} \Delta_{3 / 2}$ and ${ }^{2} \Pi_{1 / 2}$ states with energies of $72.219 \mathrm{eV}$ and $72.538 \mathrm{eV}$, respectively. These ionization energies for the ligand-field split states of $\mathrm{XeF}_{2}$ are given in Table I.

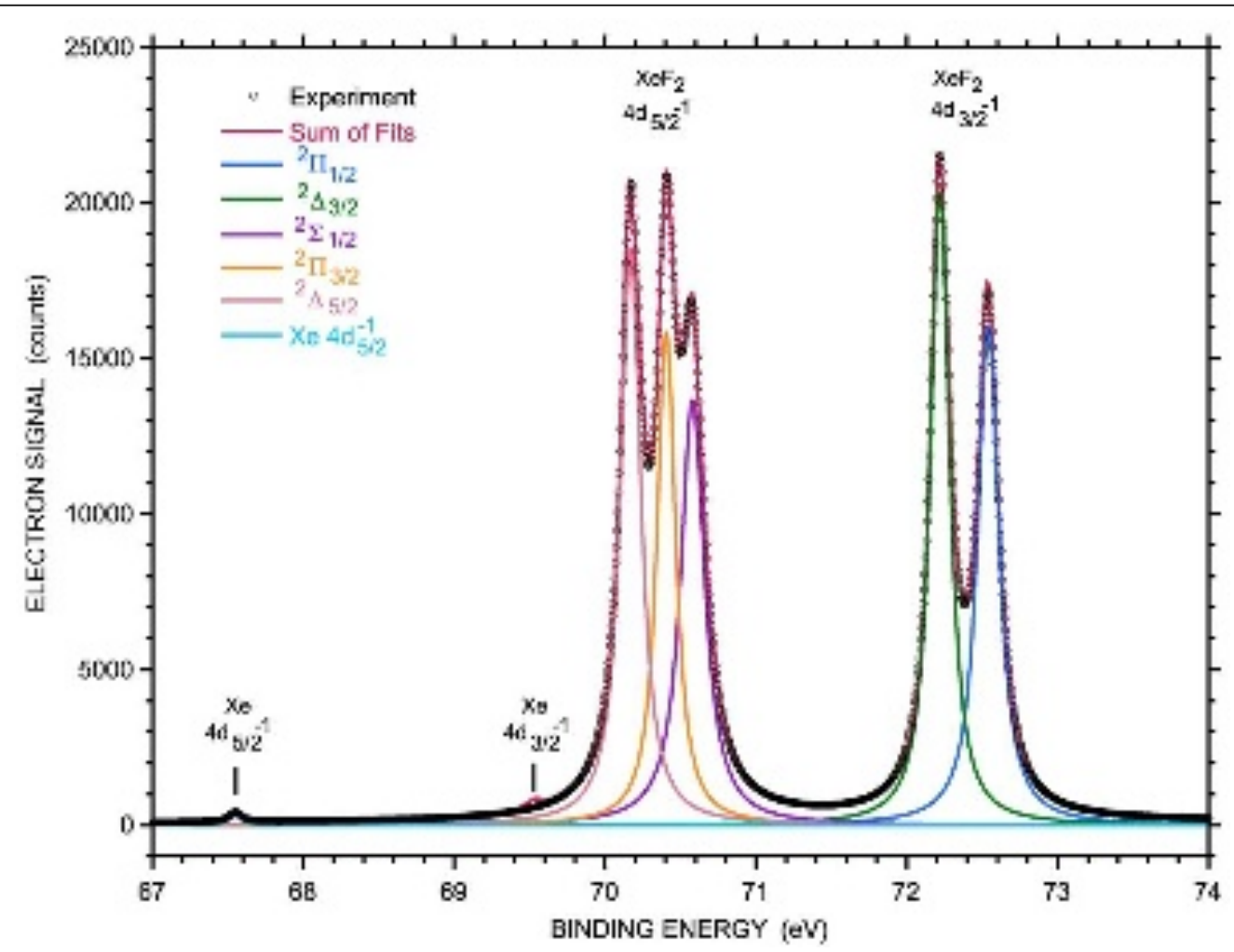

Figure 1. The photoelectron spectrum of the $4 \mathrm{~d}^{-1}$ features recorded at $115 \mathrm{eV}$ with $\theta=0^{\circ}$, along with the results of the fitting procedure.

\section{A. Ion Yield Spectra and Partial Cross Sections}

Figure 2a shows the ion yield curve between $60 \mathrm{eV}$ and $74 \mathrm{eV}$, that is, from approximately ten $\mathrm{eV}$ below the $\mathrm{XeF}_{2} 4 \mathrm{~d}$ thresholds to a few $\mathrm{eV}$ above them. Figure $2 \mathrm{~b}$ shows a detail of the structure between $67 \mathrm{eV}$ and $72 \mathrm{eV}$. In Figures $2 \mathrm{a}$ and $2 \mathrm{~b}$, the ion counts were normalized to the photodiode current. The energy scale of the ion yield was calibrated by using the energy of $65.110 \mathrm{eV}$ for the $\mathrm{Xe} 4 \mathrm{~d}_{5} / 2 \rightarrow 6 \mathrm{p}$ transition. ${ }^{5}$ The spectrum is quite similar to the photoabsorption spectrum of Comes et al., ${ }^{31}$ with some small $(<0.2 \mathrm{eV})$ differences in the resonance positions, 
but the higher resolution $(\sim 4 \mathrm{meV})$ of the present spectrum reveals a splitting of some of the features and allows the observation of more members of the Rydberg series. Note that because Figure 2 and Figure 3 (below) correspond to the total ion yield, some differences with the photoabsorption spectrum are expected. In particular, photoionization followed by Auger decay

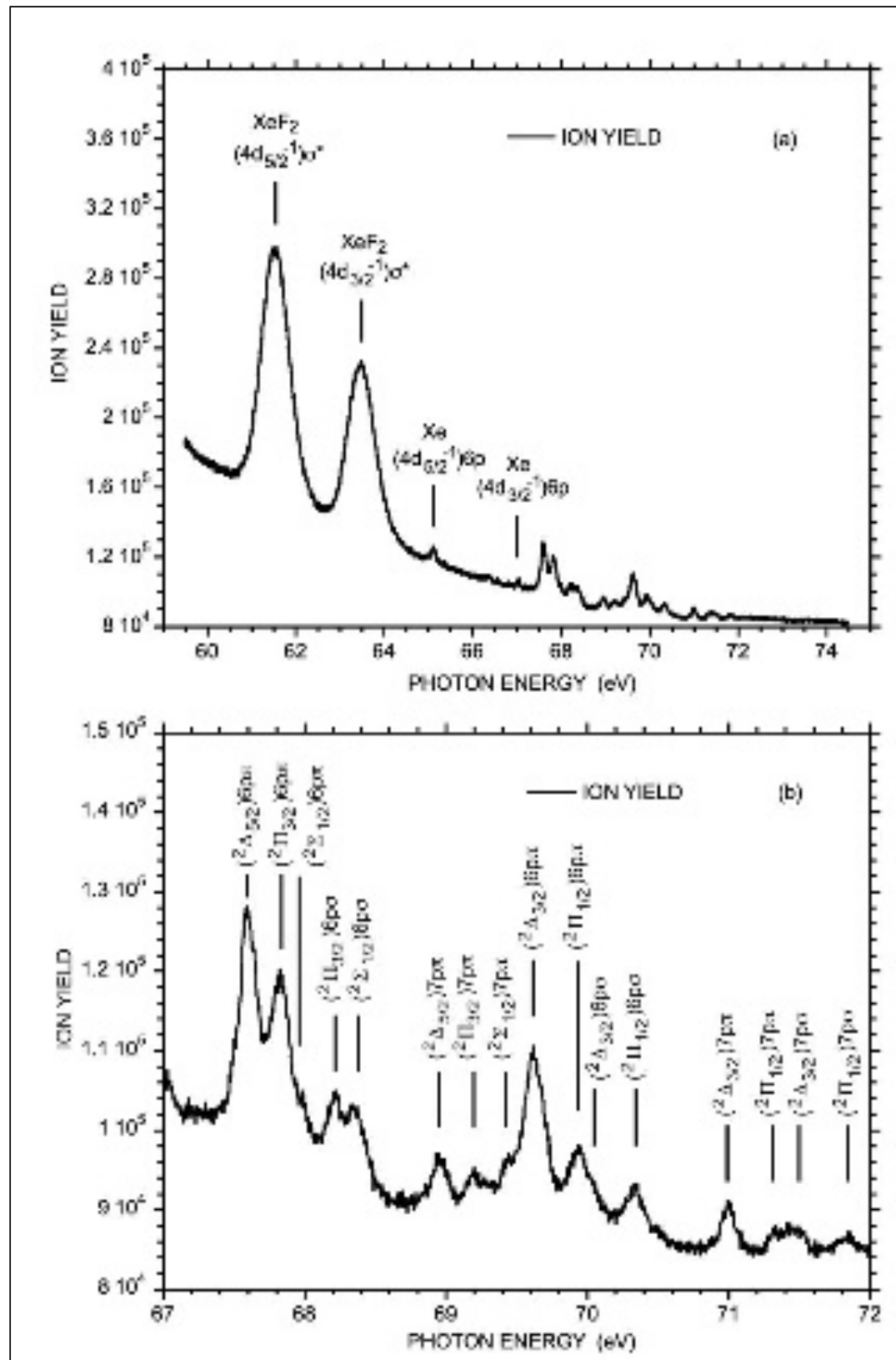

Figure 2. (a) The total ion yield following the photoionization

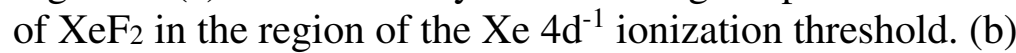
An expanded version of the same yield highlighting the region of resonance structures between photon energies of 67 and 72 $\mathrm{eV}$. or direct double

photoionization produces

doubly or multiply charged

ions that can fragment to

produce multiple counts

following the absorption of a

single photon. This issue is

more significant above the $\mathrm{Xe}$

$4 p^{-1}$ threshold, where direct

double ionization can be

substantial. ${ }^{61}$ Nevertheless,

the overall shape of the

present ion yield curve

matches the shape of the

absorption spectrum quite

well.

The two lowest energy

features in Figure 2a

correspond to transitions from

the $4 \mathrm{~d}$ shell into the

unoccupied $\sigma_{\mathrm{u}}{ }^{*}$ valence

orbital, which Comes et al. ${ }^{31}$ 
refer to as "5p". In their model, the fluorine atoms withdraw approximately one electron each from the atomic Xe 5p orbital, leaving a partially occupied orbital into which the $4 \mathrm{~d}$ electron can be excited. The two resonances are separated by $1.929 \mathrm{eV}$, very close to the $2.01 \mathrm{eV}$ spin-orbit splitting of the $\mathrm{XeF}_{2} 4 \mathrm{~d}_{5 / 2}-4 \mathrm{~d}_{3 / 2}$ multiplets, but the features are so broad that the ligand-field splittings are not observable. At higher energy, Figure 2 shows a number of weaker features, which Comes et al. ${ }^{31}$ analyzed in terms of Rydberg series converging to the five ligand- and spin-orbit split $\mathrm{XeF}_{2} 4 \mathrm{~d}^{-1}$ thresholds. For excitation from the $4 \mathrm{~d}$ shell, the dominant transitions are expected to be to the np $\sigma$ and np $\pi$ Rydberg states based on these five thresholds. Comes et al. ${ }^{31}$ argue that because electrons on the $\mathrm{F}$ atoms are more effective at screening the po electrons than the $\mathrm{p} \pi$ electrons from the $\mathrm{Xe}^{+}$core, the $\mathrm{p} \pi$ orbitals are expected to be more strongly bound. Excitation from the $4 \mathrm{~d}$ orbital into the $\mathrm{f}$ Rydberg series is weak due to the centrifugal barrier that keeps the $f$ electron at long range. At higher energies, the $f$ electron penetrates the barrier, giving rise to the well-known Xe 4d-ef shape resonance in the continuum. ${ }^{7}$

The np $\sigma$ and np $\pi$ Rydberg levels can be denoted $\left[{ }^{2} \Lambda^{+}{ }_{\Omega^{+}}\right] \mathrm{np} \lambda_{\Omega \mathrm{e}}, \Omega^{\prime}$, where the quantity in brackets describes the $4 \mathrm{~d}^{-1}$ core level, and $\Omega^{\prime}$ is the sum of the molecular frame projections $\Lambda^{\prime}$ and $\Sigma^{\prime}$. The spin splitting resulting from the coupling of $\Omega^{+}$and $\Omega_{\mathrm{e}}$ is small and not resolved in the present experiments. The electric dipole selection rule from the $\Omega^{\prime \prime}=0$ ground state of $\mathrm{XeF}_{2}$ is $\Omega^{\prime}-\Omega^{\prime \prime}=$ $0, \pm 1$. Note that for the $\left[{ }^{2} \Delta_{5 / 2}\right]$ np $\sigma_{1 / 2}$ configuration, $\Omega^{\prime}=2$ or 3 , both of which are forbidden from the ground state.

The model presented by Comes et al. ${ }^{31}$ reproduces both the splittings and intensities of the $6 \mathrm{p} \sigma$ and $6 \mathrm{p} \pi$ states reasonably well, and the same pattern of levels is observed in the new $n=7$ states observed in the present data. As expected, however, the $7 \mathrm{p} \pi-7 \mathrm{p} \sigma$ splitting is considerably smaller than the $6 \mathrm{p} \pi-6 \mathrm{p} \sigma$ splitting as a result of the $1 / \mathrm{n}^{* 3}$ scaling with effective principal quantum number, $n *$. Table II gives the observed transition energies, assignments based on the work of Comes et al., ${ }^{31}$ and effective principal quantum numbers calculated by using: 


$$
\left.\mathrm{n}^{*}=\sqrt{R y /(I E \quad E}\right),
$$

where Ry is the Rydberg constant for $\mathrm{XeF}_{2}(13.6056 \mathrm{eV}), \mathrm{IE}$ is the ionization energy, and $\mathrm{E}$ is the energy of the level. The $n^{*}$ values can be calculated with respect to each ligand-field split threshold, and the relevant values are given in Table II. The valence shell configuration of $\mathrm{XeF}_{2}$ is given in Table III.

While the improved resolution of the present experiments allows the observation of additional features, the only real difference in the assignments compared with those of Comes et al. ${ }^{31}$ is that they assign two weak features just below the corresponding spin-orbit split $6 \mathrm{p} \pi$ features to transitions to the $6 \mathrm{~s}$ Rydberg states converging to $\mathrm{XeF}_{2} 4 \mathrm{~d}^{-1}$. The $4 \mathrm{~d} \rightarrow 6$ s transitions are

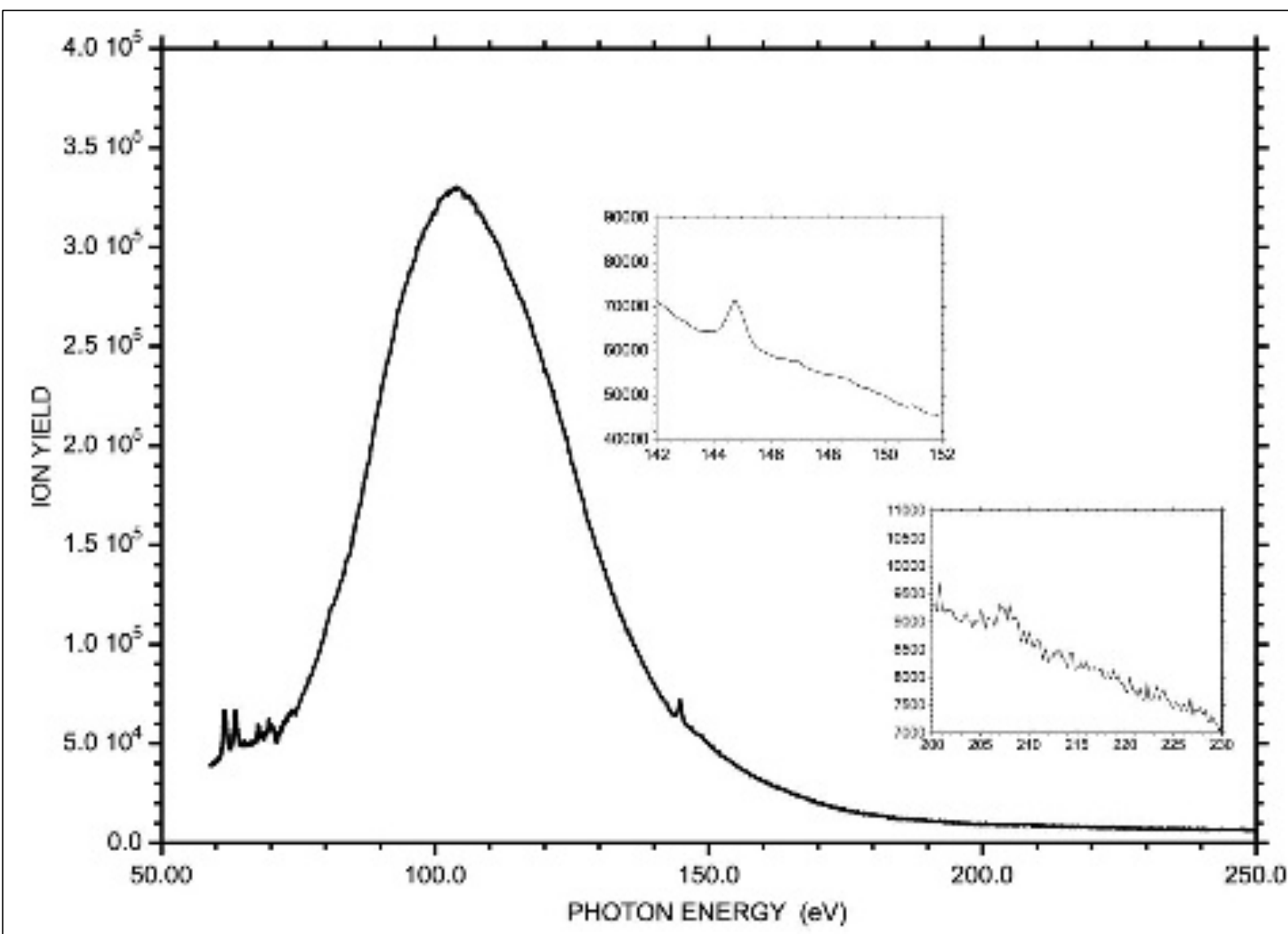

Figure 3. The total ion yield curve following the photoionization of $\mathrm{XeF}_{2}$ for photon energies between 60 and $250 \mathrm{eV}$. The insets show expanded versions of the resonance structures in the regions around the $\mathrm{Xe} 4 \mathrm{p}$ and 4 s ionization thresholds. 
expected to be forbidden, but Comes et al. suggest that they become allowed by vibronic couplings. In the present spectrum, we do see the analogous features, but assign them to different final states. In particular, the feature at 67.028 is assigned as atomic $\mathrm{Xe}\left(4 \mathrm{~d}_{3 / 2}{ }^{-1}\right) 6 \mathrm{p}$, and the feature at 68.961 is assigned as $\mathrm{XeF}_{2}\left({ }^{2} \Delta_{5 / 2}\right) 7 \mathrm{p} \pi$.

Figure 3 shows the total ion yield spectrum across a larger energy range from $\sim 60 \mathrm{eV}$ to $\sim 250$ $\mathrm{eV}$. Unfortunately, this total ion yield is not normalized to the incident photon intensity because over this extended energy range the photodiode signal exhibited prominent structure due to the $\mathrm{Si}$ edges and to contamination. The spectrum is dominated by the $4 \mathrm{~d} \rightarrow \varepsilon f$ shape resonance, and the overall appearance is very similar to that of atomic Xe, with only small differences in the peak positions and resonance width. Thus, the molecular field does not appear to have a strong effect on the ion yield from the $4 \mathrm{~d}$ shell. In addition to the sharp resonances below $70 \mathrm{eV}$, the spectrum shows weak sharp features in the two regions shown as insets in Figure 3. First, a small sharp feature is observed at 144.8(2) eV, with some evidence for very weak oscillations just above it. This feature is the molecular analog of the Xe $4 \mathrm{p} \rightarrow 6$ s transition observed by Codling and Madden $^{62}$ at $141.961 \mathrm{eV}$. As in the case of the $4 \mathrm{~d}$ resonances, the position is shifted to higher energy in $\mathrm{XeF}_{2}$ due to the electron withdrawing characteristics of the $\mathrm{F}$ atoms. The second weak feature is observed at 207.8(4) eV, as compared to the $\mathrm{XeF}_{2} 4 \mathrm{~s}^{-1}$ threshold at $216.04 \pm 0.10 \mathrm{eV}^{33}$ yielding a binding energy of $\sim 8.24 \mathrm{eV}$. Based on the binding energy of the $\sigma_{\mathrm{u}}{ }^{*}$ valence orbital with respect to the $4 \mathrm{~d}^{-1}$ threshold, the most likely assignment for the $207.8 \mathrm{eV}$ feature is $4 \mathrm{~s} \rightarrow$ $\sigma_{\mathrm{u}}^{*}$.

Figure $4 \mathrm{a}$ shows the present theoretical $4 \mathrm{~d}$ cross section for atomic Xe, along with the experimental $4 \mathrm{~d}$ cross section from Becker et al., ${ }^{13}$ and the total photoionization cross section from West and Morton. ${ }^{6}$ Theoretical calculations based on the relativistic random phase approximation (RRPA) reproduce the experimental Xe cross section with very good agreement. ${ }^{7}$ The present ePolyScat results, which were obtained using the length gauge, peak approximately 
$10 \mathrm{eV}$ below the

experimental energy, and

the calculated resonance

width is only $\sim 60 \%$ of that

for the experiment. The

cross section calculated

using the velocity gauge

has a similar shape, but the

magnitude is $\sim 8$ times

larger at its peak; this

difference may be

indicative of correlation

effects. The differences

between the present results

and the more accurate

results are attributed

primarily to the lack of

treatment of many body

effects in the present

calculation, as noted in

Section 2.C, ${ }^{57}$ although

computational factors such

as the exact form of the

scattering potential will

also play a role.
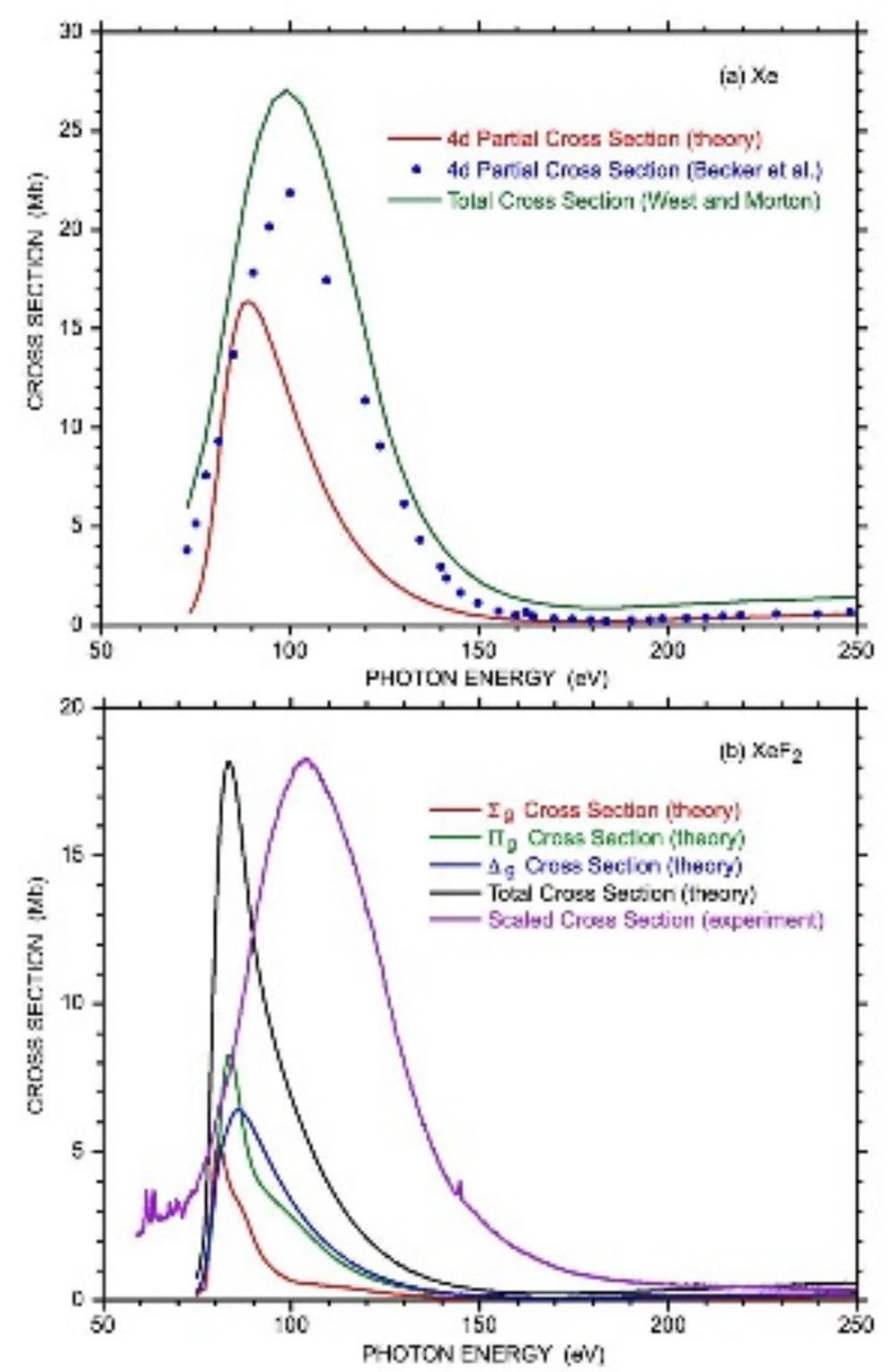

Figure 4. (a) The present theoretical $4 d$ partial cross section for atomic Xe, along with the experimental $4 d$ photoionization cross section from Becker et al. (Ref. 13), and the total photoionization cross section from West and Morton (Ref. 6). (b) A comparison of the theoretical 4d cross section for $\mathrm{XeF}_{2}$ and the present experimental data from Figure 2. The theoretical ligand-field-split partial cross sections are also shown in the absence of spin-orbit coupling. 
Figure $4 \mathrm{~b}$ shows a comparison of the calculated $4 d$ cross section for $\mathrm{XeF}_{2}$ with the experimental data from Figure 3. The experimental ion yield is not absolute, and it has been scaled to the maximum of the theoretical spectrum. Theoretical cross sections for the individual ligand-field split continua are also shown in the absence of spin-orbit coupling. The level of agreement is comparable to that for atomic $\mathrm{Xe}$, with the theoretical cross section peaking at lower energy, and displaying a considerably smaller width than the experimental curve. Interestingly, prior to vibrational averaging the theoretical cross sections also showed a number of continuum resonances between 80 and $90 \mathrm{eV}$ that are not evident in the experimental cross section, as well as more complex low-energy behavior. Many of these features are, however, washed out in the vibrationally-averaged results shown here. In earlier studies of the valence shell ionization of $\mathrm{XeF}_{2}$, Yates et al. ${ }^{63}$ and $\mathrm{Tse}^{64}$ found strong resonant features $10-15 \mathrm{eV}$ above the threshold for photoionization out of the $10 \sigma_{\mathrm{g}}$ and $3 \pi_{\mathrm{g}}$ orbitals. Tse interpreted these resonances as excitation from the F-dominated $10 \sigma_{\mathrm{g}}$ and $3 \pi_{\mathrm{g}}$ orbitals into the continuum dominated by Xe $\varepsilon f$ character ${ }^{64}$ In the present case, excitation occurs from the $\mathrm{Xe} 4 \mathrm{~d}$ orbital, but is likely into the
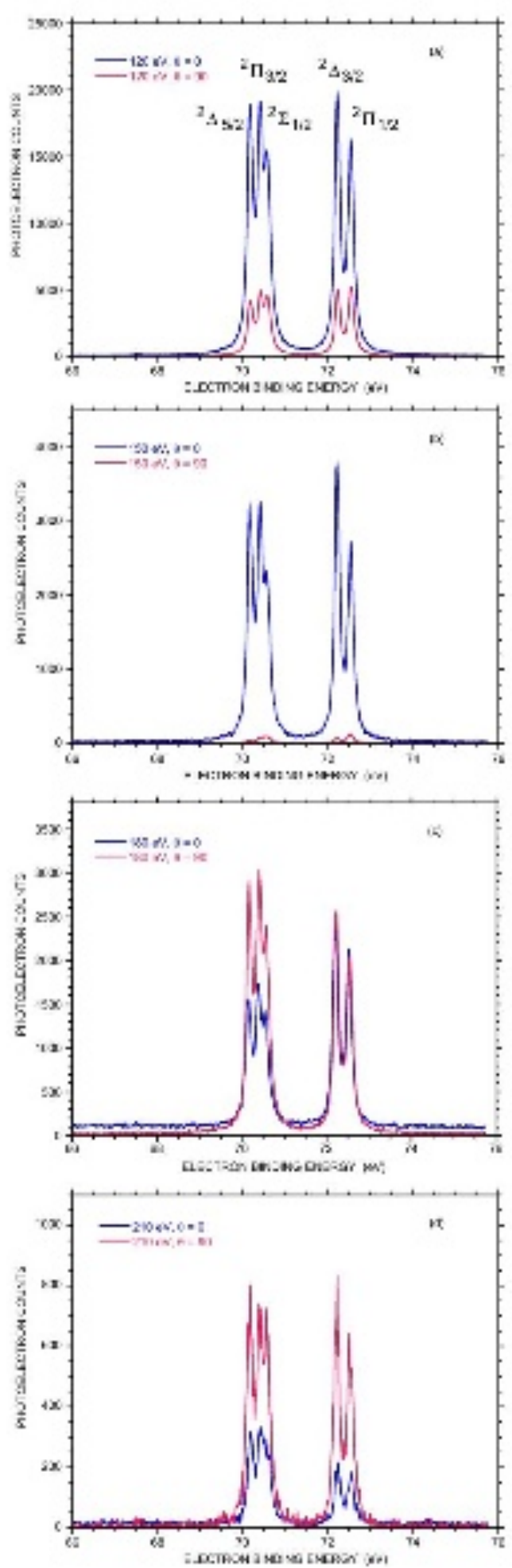

Figure 5. Representative photoelectron spectra of the $4 \mathrm{~d}^{-1}$ features recorded (a) $120 \mathrm{eV}$, (b) $150 \mathrm{eV}$, (c) $180 \mathrm{eV}$, and (d) $210 \mathrm{eV}$ with $\theta=0^{\circ}$ and $90^{\circ}$. 


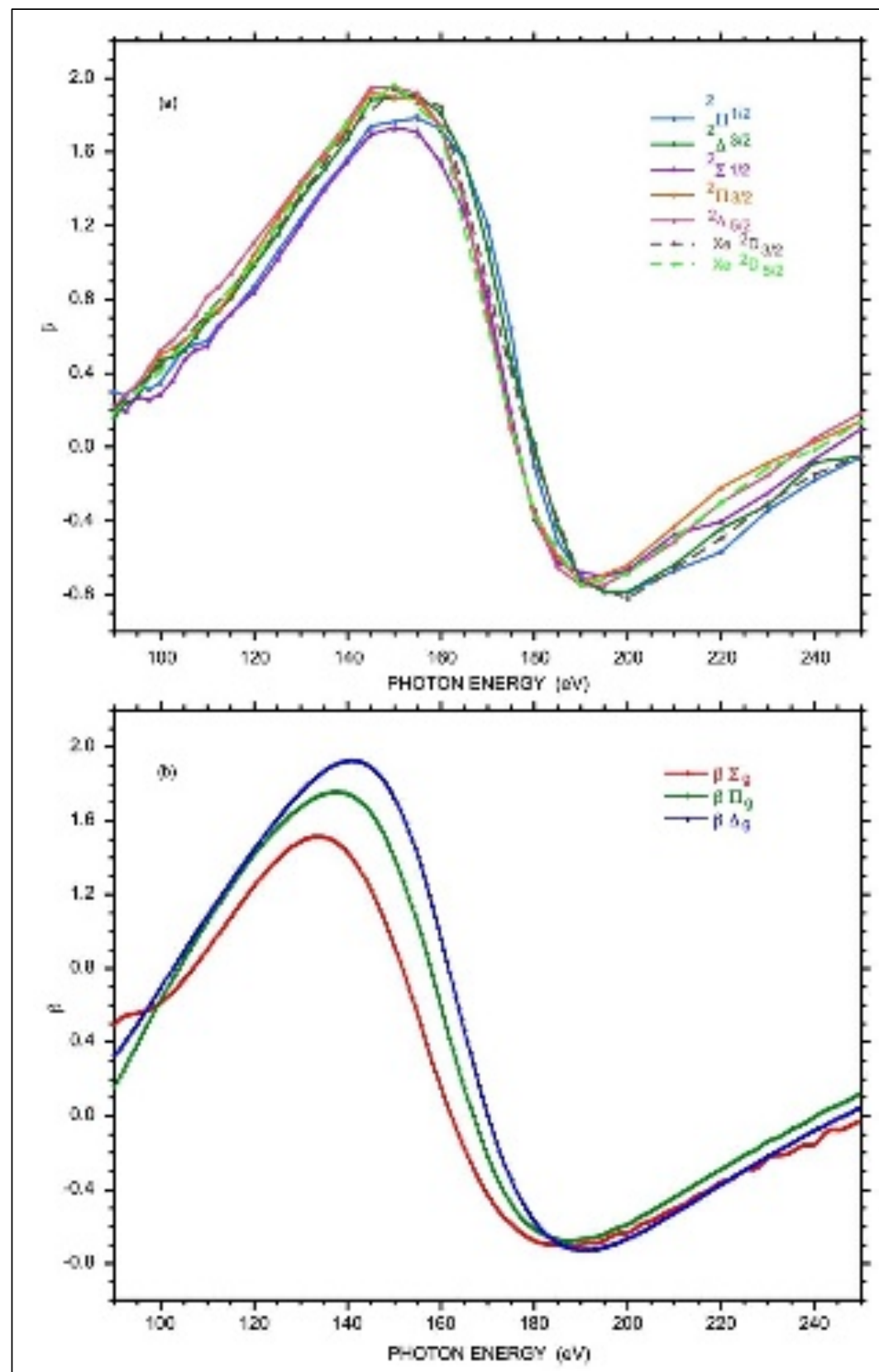

Figure 6. (a) The substate-resolved $\beta$ parameters for the photoionization of $\mathrm{XeF}_{2}$ between 95 and $250 \mathrm{eV}$, along with the corresponding spin-orbit resolved data on atomic Xe. (b) The theoretical ligand-field resolved $\beta$ curves in the absence of spin-orbit coupling for photoionization from the $4 \mathrm{~d}$ shell of $\mathrm{XeF}_{2}$. analogous continuum resonances. Further details of these features remain a topic for future work, and numerical artefacts have not been fully ruled out. No such structures have been observed in the current experimental results.

We have recorded the photoelectron spectra for the $4 \mathrm{~d}^{-1}$ photolines of $\mathrm{XeF}_{2}$ from threshold to $250 \mathrm{eV}$ with sufficient resolution to resolve the ligand-field and spin-orbit substates. Figure 5 shows examples of the photoelectron spectra recorded at photon energies of $120,150,180$, and 210 $\mathrm{eV}$, for radiation polarized normal to the beam direction and in the planes parallel and

perpendicular to the entrance axis of the electron spectrometer. These fits allow the determination of the photoelectron angular distribution parameter, $\beta$, as well as the branching fractions for the different substates. We note that, below approximately $95 \mathrm{eV}$ photon energy, 
post-collision interaction effects resulting from the interaction between the photoelectrons and the Auger electrons can broaden and distort the lineshapes. ${ }^{65,66,67}$ We have not modeled these effects here, and focus on the data between 95 and $250 \mathrm{eV}$.

Figure 6 a shows the experimental substate-resolved $\beta$ parameters for the $4 \mathrm{~d}^{-1}$ photoionization of $\mathrm{XeF}_{2}$ between 90 and $250 \mathrm{eV}$, along with the corresponding spin-orbit resolved data for $\mathrm{Xe}$. Measurements for atomic Xe have been reported previously, ${ }^{10,23}$ and the energy dependence of the $\beta$ curves is well reproduced by theory, ${ }^{23,68}$ with the dramatic profile resulting from the shape resonance in the $\mathrm{f}$ continuum. The curves for the $\mathrm{Xe}^{+} 4 \mathrm{~d}_{5 / 2}$ and $4 \mathrm{~d}_{3 / 2}$ final states are shifted slightly from each other, with some subtle differences in shape. As discussed by Wang et al., ${ }^{23}$ in the absence of dynamical effects resulting from relativistic interactions, the $\beta$ parameters for the two substates should be identical when plotted vs. electron kinetic energy. (When plotted vs. photon energy, the $4 \mathrm{~d}_{3 / 2}$ curve is shifted $\sim 2 \mathrm{eV}$ above the $4 \mathrm{~d}_{5 / 2}$ curve.) The differences they observed could be explained with their calculations using the relativistic random phase approximation. ${ }^{23}$ The present data for Xe are consistent with their measurements, but ours were not performed on as fine an energy grid.

The substate-resolved $\beta$ values for $\mathrm{XeF}_{2}$ in Figure $6 \mathrm{a}$ are all quite similar, both to each other and to the atomic Xe data. Two small differences deserve mention. First, below $\sim 160 \mathrm{eV}$, the $\beta$ values in Figure 6a for the $4 \mathrm{~d}_{5 / 2}{ }^{2} \Sigma_{1 / 2}$ and $4 \mathrm{~d}_{3 / 2}{ }^{2} \Pi_{1 / 2}$ substates lie noticeably below the corresponding values for the other substates. Second, above $\sim 190 \mathrm{eV}$, the $\beta$ values for the $4 \mathrm{~d}_{3 / 2}$ ${ }^{2} \Pi_{1 / 2}$ and ${ }^{2} \Delta_{3 / 2}$ substates lie significantly below the other curves, and they are similar to the Xe ${ }^{2} \mathrm{D}_{3 / 2}$ curve. This higher energy region is also where the $\mathrm{Xe}^{+} 4 \mathrm{~d}_{3 / 2} \beta$ curve differed more substantially from the $4 \mathrm{~d}_{5 / 2}$ curve, an observation that can also be made in Figure 6a. The present angular distribution parameters do not appear to be affected very strongly by the resonances associated with photoionization from the $4 \mathrm{p}$ and $4 \mathrm{~s}$ shells, but this might simply reflect the low intensities of the resonances and the coarseness of the energy grid in Figure 6a. 
Kukk et al. ${ }^{69}$ have performed a similar study of the molecular-field and spin-orbit resolved substates of $\mathrm{OCS}^{+}$populated by $\mathrm{S} 2 \mathrm{p}$ photoionization. They observed significant differences in their substate-resolved angular distribution parameters relative to those given by atomic-like calculations. These differences are greatest close to threshold, and reflect the molecular character of the system. At higher photoelectron energies, where the dynamics are much less sensitive to molecular effects, these effects become much smaller. The present $\mathrm{XeF}_{2}$ data are consistent with the latter observation, with the molecular curves converging to the corresponding $\mathrm{Xe}^{+}$spin-orbit state curves at high energy. Unfortunately, in the present experiment, the region close to threshold is complicated by a number of factors including shape resonances and post-collisioninteraction (PCI) effects that make it difficult to calculate the molecular-field and spin-orbit dependent behavior. In addition, PCI effects will depend on the photoelectron and Augerelectron angular distributions, which will be modified by resonance effects in different channels, requiring theoretical analysis beyond the scope of the present paper.

In atomic $\mathrm{Xe}$, there is a Cooper minimum in the $4 \mathrm{~d} \rightarrow \varepsilon \mathrm{f}$ cross section that occurs at a photon energy of $\sim 185(10) \mathrm{eV},{ }^{10,23}$ and this minimum is also expected in the $\mathrm{XeF}_{2} 4 \mathrm{~d} \rightarrow$ cf cross section. For an ionization channel generally, the observed minima in the cross section and $\beta$ parameter may differ, and neither may correspond to the actual energy at which a radial dipole matrix element vanishes (this being the quantum definition for a Cooper minimum ${ }^{70}$ ) if the other nonzero matrix elements are energy dependent. Wang et al., ${ }^{23}$ have used the non-relativistic CooperZare formula ${ }^{71}$ for $\beta$ in the $4 \mathrm{~d} \rightarrow \varepsilon \mathrm{p}$, $\varepsilon f$ ionization to predict that, as the $4 \mathrm{~d} \rightarrow \varepsilon \mathrm{f}$ matrix element goes through zero, the photoelectron angular distribution would have $\beta=0.2$. This equality then provides an alternative means to identify a precise energy of the Cooper minimum from a plot of the $\beta$ parameter. In the relativistic case, the situation is more complicated because there are multiple continua with matrix elements that pass through zero at slightly different energies (e.g., $4 \mathrm{~d}_{5 / 2} \rightarrow \varepsilon \mathrm{f}_{5 / 2}$ and $\left.\varepsilon \mathrm{f}_{7 / 2}\right)$. Nevertheless, Wang et al. ${ }^{23}$ found that the $\beta$ value corresponds to 0.2 at 
the average position of the minima in the two channels, and that the $\beta_{5 / 2}$ value was 0.2 at about 2 $\mathrm{eV}$ lower electron kinetic energy ( $4 \mathrm{eV}$ lower in photon energy) than the $\beta_{3 / 2}$ value. In the present $\mathrm{Xe}$ data, the $\beta_{5 / 2}$ and $\beta_{3 / 2}$ values are 0.2 at photon energies of $174.5 \mathrm{eV}$ and $177.3 \mathrm{eV}$, respectively (corresponding to a $0.8 \mathrm{eV}$ difference in electron kinetic energy). The present values are consistent with the determinations of Lindle et al. ${ }^{10}$ and Wang et al. ${ }^{23}$ and are slightly lower than the position of the minimum in the total photoionization cross section $(180-185 \mathrm{eV}){ }^{6}$

In $\mathrm{XeF}_{2}$, the situation is somewhat more complicated than in $\mathrm{Xe}$ because there are both spin-orbit and ligand-field splittings, and because the minima in all of the $4 \mathrm{~d} \rightarrow \varepsilon f \sigma, \varepsilon f \pi, \varepsilon f \delta$, and $\varepsilon f \phi$ matrix elements may occur at different energies. However, the similarities of the $\mathrm{XeF}_{2}$ and $\mathrm{Xe}$ data suggest that these differences are rather small. As seen in Figure $6 \mathrm{a}$, the $\mathrm{XeF}_{2} 4 \mathrm{~d}_{5 / 2}{ }^{-1}{ }^{2} \Sigma_{1 / 2}$, ${ }^{2} \Pi_{3 / 2}$, and ${ }^{2} \Delta_{5 / 2} \beta$ values reach a value of 0.2 at a photon energy of $174.5 \mathrm{eV}$, the same photon energy as the $\mathrm{Xe} 4 \mathrm{~d}_{5 / 2}{ }^{-1} \beta$ value. Similarly, the $\mathrm{XeF}_{2} 4 \mathrm{~d}_{5 / 2}{ }^{-1}{ }^{2} \Pi_{1 / 2}$, and ${ }^{2} \Delta_{3 / 2} \beta$ values reach 0.2 at $177.3 \mathrm{eV}$, as does the $\mathrm{Xe} 4 \mathrm{~d}_{3 / 2^{-1}}$ value. The $\mathrm{XeF}_{2}$ values correspond to electron kinetic energies that are $\sim 2.9 \mathrm{eV}$ smaller than the corresponding Xe values. This difference implies the electron withdrawing nature of the $\mathrm{F}$ atoms results in a slightly different energy dependence of the $4 \mathrm{~d} \rightarrow$ ef transition matrix elements.

Figure $6 \mathrm{~b}$ shows the theoretical $\beta$ curves for $\mathrm{XeF}_{2}$ for the three ligand-field components in the absence of spin-orbit coupling. In contrast to the cross-section calculation, the agreement with the experimental curves of Figure 6a is quite good. The curves do peak at approximately 15 - 20 $\mathrm{eV}$ lower energy than the experimental ones, and their minima are shifted by a similar amount. The curve with the smallest maximum $\beta$ ( $\sum_{\mathrm{g}}$ symmetry) is the same as that in the experiment $\left({ }^{2} \Sigma_{1 / 2}\right)$, and the curves with the largest $\beta$ are also the same $\left(\Delta_{\mathrm{g}}\right.$ and $\left.{ }^{2} \Delta_{5 / 2}\right)$. The $\beta$ value in the $\Sigma_{\mathrm{g}}$ channel differs most from the atomic $\beta$ curves because the ${ }^{2} \Sigma_{1 / 2 g}$ orbital along the F-Xe-F axis is the most effected by the electron-withdrawing character of the F atoms, i.e., the molecular effects. The improved agreement for the $\beta$ values can be rationalized because this is primarily a 
single-channel response, which suggests that the scattering potential is reasonably accurate, and the scattering dynamics are described reasonably well. This observation is consistent with the previous statement (Section 2.C) that the relatively poor agreement for the cross section is likely attributed to many-body effects.

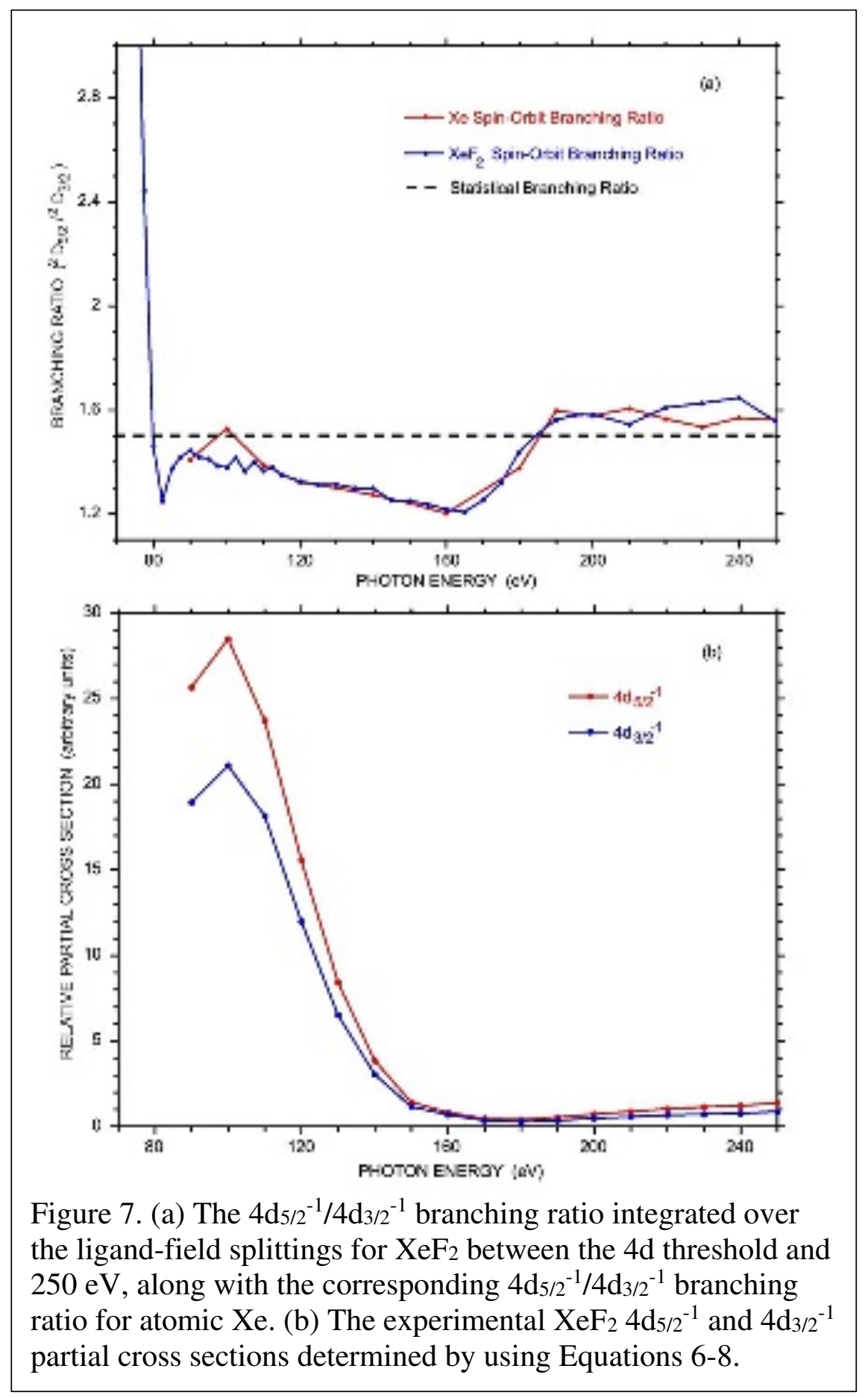

Figure 7a shows the $4 \mathrm{~d}_{5 / 2} / 4 \mathrm{~d}_{3 / 2}$ spin-orbit branching ratio for $\mathrm{XeF}_{2}$ integrated over the separate ligand-field-split states, along with the corresponding spin-orbit branching ratios for atomic Xe. The strong similarity between the two curves, even including the shallow minimum and strong rise as the photon energy approaches threshold, suggests that the spin-orbit branching ratios are largely governed by atomic effects. The detailed shape of the spin-orbit branching ratios for atomic Xe are wellreproduced by theory. ${ }^{7,14,18}$ Above $100 \mathrm{eV}$, the 
branching ratio is close to the statistical value of 1.5 , with only small excursions from this value up to $250 \mathrm{eV}$.

Figure 8 shows the ligand-field-split substate branching fractions for $\mathrm{XeF}_{2}$, where the sums of the values are separately normalized for the two spin-orbit states. For the $4 \mathrm{~d}_{3 / 2}{ }^{-1}$ component, the ${ }^{2} \Pi_{1 / 2}$ and ${ }^{2} \Delta_{3 / 2}$ branching fractions are both close to the statistical value of 0.5 , with the ${ }^{2} \Delta_{3 / 2}$

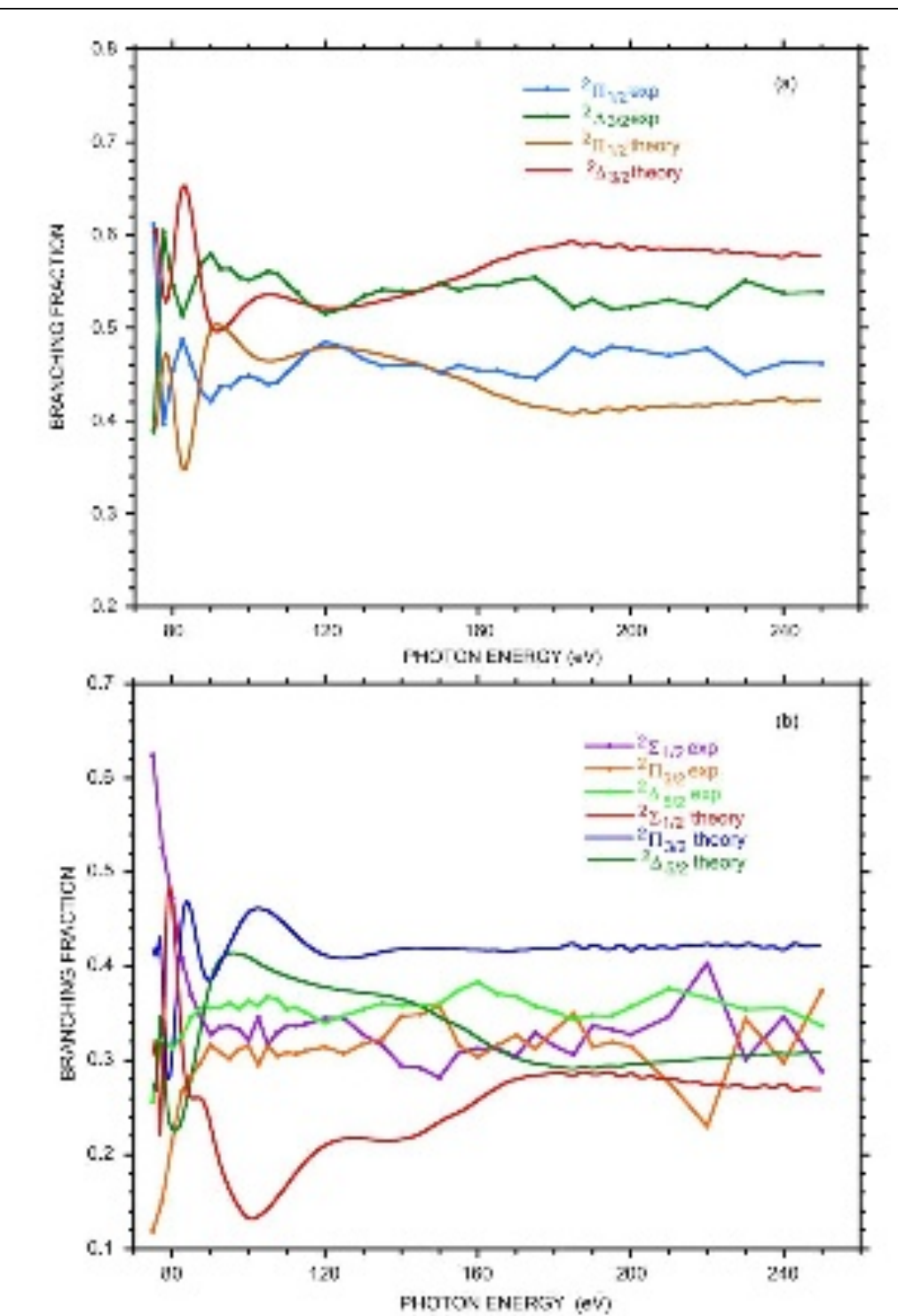

Figure 8. The experimental ligand-field and spin-orbit resolved branching fractions for $4 \mathrm{~d}$ photoionization of $\mathrm{XeF}_{2}$ between threshold and $250 \mathrm{eV}$. The corresponding theoretical branching fractions including both ligand-field and spin-orbit interactions are also shown. (a) The $4 \mathrm{~d}_{3 / 2}{ }^{-1}$ branching fractions. (b) The $4 \mathrm{~d}_{5 / 2^{-1}}$ branching fractions. fraction being slightly larger, and there are only small variations with the photon energy. Similarly, for the $4 \mathrm{~d}_{5 / 2}{ }^{-1}$ component, the ${ }^{2} \Sigma_{1 / 2},{ }^{2} \Pi_{3 / 2}$, and ${ }^{2} \Delta_{5 / 2}$ branching fractions are all approximately equal to the statistical value of 0.33. Again, only small variations are observed as a function of photon energy.

Figure 8 also shows the results of theoretical calculations using the present model for spin-orbit coupling. Overall, the agreement with experiment is quite good, particularly for the $4 \mathrm{~d}_{3 / 2}$ component. However, the calculations show rather more modulations in the branching fractions, even though these are relatively small in magnitude. 


\begin{abstract}
Although the theoretical branching fractions do show some oscillations, the lack of a stronger energy dependence in both the experimental and theoretical data seems somewhat surprising because the different ligand-field-split substates are associated with different sets of continuum partial waves, and the photoionization matrix elements show considerable variations in magnitude between 90 and $250 \mathrm{eV}$. As in the case of the $\beta$ values, the reasonably good agreement between experiment and theory suggests that the branching fractions are primarily dependent on single-channel effects that are well-described by the present wavefunctions and matrix elements.
\end{abstract}

One set of photoelectron data was recorded using a mixture of $\mathrm{Xe}$ and $\mathrm{XeF}_{2}$ to provide a simultaneous measurement of the $4 \mathrm{~d}^{-1}$ spectra of the two species. The photoelectron data recorded with parallel and perpendicular polarization can be used to synthesize a "magic angle" photoelectron spectrum $\left(\mathrm{I}_{\mathrm{m}}\right)$ by using: ${ }^{72,73}$

$$
I_{m}=\frac{\left(I_{0}+2 I_{90}\right)}{3}
$$

in which the relative intensities of the peaks reflect the relative cross sections for the different ionization channels independent of differing angular distributions.

The integrated signals in the $4 \mathrm{~d}^{-1} \mathrm{Xe}$ and $\mathrm{XeF}_{2}$ photoelectron peaks can be combined with the well-known absolute photoionization cross section of $\mathrm{Xe}^{10,13}$ and the relative concentrations of $\mathrm{Xe}$ and $\mathrm{XeF}_{2}$ to yield the absolute photoionization cross sections of $\mathrm{XeF}_{2}$ :

$$
\mathrm{XeF}_{2}(E)=\frac{N_{\mathrm{XeF}_{2}}(E)_{\mathrm{Xe}}(E)}{N_{\mathrm{Xe}}(E)}\left(\frac{[\mathrm{Xe}]}{\left[\mathrm{XeF}_{2}\right]}\right) .
$$

Unfortunately, the relative concentrations of $\mathrm{Xe}$ and $\mathrm{XeF}_{2}$ are not well-characterized, although they are expected to be relatively constant over the time of the measurement. Taking their ratio as a constant, Equation 7 then provides a relative cross section measurement for $\mathrm{XeF}_{2}$. This 
result can be further refined into $\mathrm{XeF}_{2} 4 \mathrm{~d}_{5 / 2^{-1}}$ and $4 \mathrm{~d}_{3 / 2^{-1}}$ partial cross sections by separately integrating the two spin-orbit components of the $\mathrm{XeF}_{2}$ signal and using:

$$
\mathrm{XeF}_{2}\left(4 d_{5 / 2}^{1}\right)=\frac{N_{\mathrm{XeF}_{2}}\left(4 d_{5 / 2}^{1}\right)}{N_{\mathrm{XeF}_{2}}} \quad \mathrm{XeF}_{2},
$$

and the analogous equation for the $4 \mathrm{~d}_{3 / 2}{ }^{-1}$ component. The relative partial cross sections are shown in Figure $7 \mathrm{~b}$. The Cooper minima in both channels are clearly observed near $180 \mathrm{eV}$, but the energy grid is not sufficiently fine to locate them more precisely.

\section{B. Shake-Up and Shake-Off Spectra}

Photoionization from the inner-shell orbitals is often accompanied by weaker shake-up or shakeoff processes in which a second electron is excited or ejected from a valence orbital in conjunction with the ejection of the core electron. ${ }^{74}$ Such processes typically occur in the energy region $\sim 5-25 \mathrm{eV}$ above the inner-shell threshold. Although most of the present photoelectron spectra are focused on the $4 \mathrm{~d}^{-1}$ main lines, photoelectron spectra over a much larger kinetic energy range were recorded at photon energies of 90,120 , and $150 \mathrm{eV}$ to characterize both the shake-up and Auger processes associated with the $4 \mathrm{~d}^{-1}$ photoionization of $\mathrm{XeF}_{2}$. Tse et al. ${ }^{75}$ have discussed the $\mathrm{Xe} 3 \mathrm{~d}$ and $4 \mathrm{~d}$ shake-up processes for $\mathrm{XeF}_{2}$ following ionization by $\mathrm{Al} \mathrm{K} \alpha$ radiation $(\sim 1486 \mathrm{eV})$. The spectrum was not well-resolved, but at this energy the direct/normal shake-up process is expected to dominate, and the experimental spectrum was fitted accordingly.

Figure 9 shows a portion of the photoelectron spectrum of $\mathrm{XeF}_{2}$ recorded at $150 \mathrm{eV}$ in the region of the $4 \mathrm{~d}^{-1}$ shake-up and shake-off processes. The full spectrum is given in the Supplemental Material. The spectrum is plotted in terms of the excitation energy relative to the lowest Xe $4 \mathrm{~d}_{5 / 2}{ }^{-1}$ binding energy of $\mathrm{XeF}_{2}(70.169 \mathrm{eV})$. Also shown is the corresponding spectrum of atomic Xe recorded by Holland et al. ${ }^{76}$ and plotted relative to the atomic Xe $4 \mathrm{~d}_{5} / 2^{-1}$ binding energy 
$(67.548 \mathrm{eV}) .{ }^{5}$ Ausmees et al. ${ }^{19}$ have discussed the assignment of the latter spectrum, breaking it down into sections. The weak structure at binding energies below $16 \mathrm{eV}$ is not present in the spectrum recorded at a photon energy of $1487 \mathrm{eV}$, and it was assigned to conjugate shake-up processes to final states with $\left(4 \mathrm{~d}^{-1} 5 \mathrm{p}^{-1}\right) 5 \mathrm{~d}$ and $6 \mathrm{~s}$ configurations. The strong peaks at excitation energies of 16 to $20 \mathrm{eV}$ were assigned to the normal shake-up processes to $\left(4 \mathrm{~d}^{-1} 5 \mathrm{p}^{-1}\right) 6 \mathrm{p}$ states, and at higher energy some overlap with $\left(4 \mathrm{~d}^{-1} 5 \mathrm{p}^{-1}\right) 7 \mathrm{p}$ states is expected. Unfortunately, the spectrum is potentially very complex because the $\left(4 \mathrm{~d}^{-1} 5 \mathrm{p}^{-1}\right) \mathrm{np}$ configuration gives rise to a large number of possible states, and no detailed assignment has been given for the observed structure. The assignment of the shake-up states for $\mathrm{XeF}_{2}$ is expected to be even more challenging, although it appears that at least some features can be tentatively assigned. In particular, the $\mathrm{XeF}_{2}$ $4 \mathrm{~d}^{-1}$ shake-up spectrum shows relatively intense structure at much lower excitation energy than atomic Xe. This structure almost certainly arises from shake-up into the unoccupied $\sigma^{*}$ valence orbital, which is not present in atomic Xe.

The excitation energy of the lowest-lying feature in Figure 9 occurs at an energy of $\sim 8 \mathrm{eV}$ relative to $\mathrm{XeF}_{2}\left(4 \mathrm{~d}_{5 / 2}{ }^{-1}{ }^{2} \Delta / 2\right)$, slightly higher than the excitation energy of the corresponding $\sigma_{\mathrm{u}}{ }^{*}$ resonance in neutral $\mathrm{XeF}_{2}(7.85 \mathrm{eV}) .{ }^{77}$ Consideration of the selection rules provides insight into the nature of the shake-up process. In particular, Table III shows two mechanisms for shake-up involving the $\sigma_{\mathrm{u}} *$ resonance, along with the valence-shell configuration of $\mathrm{XeF}_{2}$. In this simple picture, configurations with multiple excitations, as well as vibronic interactions, are ignored. In the normal shake-up process, the $4 \mathrm{~d}$ electron is excited into the $\varepsilon \ell_{\mathrm{u}}$ continuum via a dipole process, and a valence electron is excited into the $\sigma_{\mathrm{u}}{ }^{*}$ orbital via a monopole transition. As seen in Table III, the only allowed monopole process is from the $6 \sigma_{u}$ orbital, which would require approximately $5 \mathrm{eV}$ more energy than excitation from the $5 \pi_{\mathrm{u}}$ highest-occupied orbital (HOMO). 


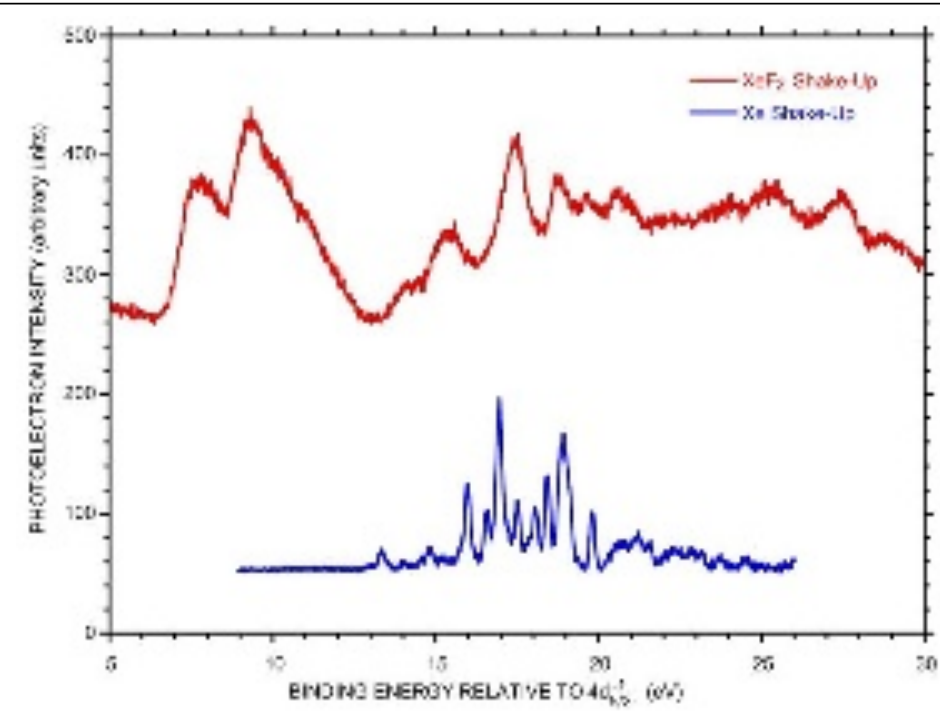

Figure 9. A portion of the $150 \mathrm{eV}$ photoelectron spectrum showing the shake-up region and plotted in terms of the excitation energy relative to the lowest $\mathrm{Xe}$ $4 \mathrm{~d}_{5} / 2^{-1}$ binding energy of $\mathrm{XeF}_{2}\left({ }^{2} \Delta 5 / 2\right.$ at $\left.70.169 \mathrm{eV}\right)$. Also shown is the corresponding spectrum of atomic Xe from Ref. 73 and plotted relative to the atomic Xe $4 \mathrm{~d}_{5} / 2^{-1}$ binding energy $(67.548 \mathrm{eV})$.
On the other hand, the conjugate process involving dipole excitation from $4 \mathrm{~d}$ to $\sigma_{\mathrm{u}}{ }^{*}$ and monopole ionization of a valence orbital is allowed for all of the valence orbitals, and is likely the dominant shake-up mechanism for these low-energy features. Alternatively, the broad shoulder and peak observed at 14.31 and $15.64 \mathrm{eV}$ excitation energy may result from the normal shake-up process involving the $6 \sigma_{u}$ orbital.

Assignments of the $\mathrm{XeF}_{2}$ shake-up features above $13 \mathrm{eV}$ in Figure 9 run into the same difficulties as for Xe, and they must await more detailed theoretical investigations. By analogy with Xe, however, it appears likely that the structure at binding energies of 13 to $\sim 20 \mathrm{eV}$ in Figure 9 are associated with $\mathrm{XeF}_{2}\left(4 \mathrm{~d}^{-1} 5 \mathrm{p}^{-1}\right) 6 \mathrm{p}$ and $7 \mathrm{p}$ excitation, and that the structure observed between 23 and $28 \mathrm{eV}$ is associated with the $\mathrm{XeF}_{2}\left(4 \mathrm{~d}^{-1} 5 \mathrm{~s}^{-1}\right)$ ns excitations. ${ }^{11}$

\section{Auger Decay Widths}

Returning to the $4 \mathrm{~d}^{-1}$ photolines, the core-excited states of $\mathrm{Xe}^{+}$resulting from the removal of a $4 \mathrm{~d}$ electron have sufficient internal energy to undergo single- or double-Auger processes ${ }^{78}$ to produce $\mathrm{Xe}^{2+}$ or $\mathrm{Xe}^{3+}$, and the situation is expected to be similar for $\mathrm{XeF}_{2}$. We now focus on the decay processes of the core-excited $\mathrm{XeF}_{2}{ }^{+}$through the consideration of the total decay rate and the Auger-electron spectrum. For the latter, we focus on the single Auger processes because the double Auger process is expected to produce slow electrons with energies below $10 \mathrm{eV}$. 
As discussed in Section II.B, the decay widths for the ligand-field- and spin-orbit-split $4 \mathrm{~d}^{-1}$ states of $\mathrm{XeF}_{2}$ can be extracted from the widths of the corresponding photoelectron peaks. In atomic $\mathrm{Xe}$, the $4 \mathrm{~d}_{5 / 2^{-1}}$ and $4 \mathrm{~d}_{3 / 2^{-1}}$ widths differ slightly, with the former being slightly larger than the latter $\left(111 \pm 3 \mathrm{meV}\right.$ vs $104 \pm 3 \mathrm{meV}$, respectively). ${ }^{22}$ Cutler et al..$^{36}$ have reported a corresponding substate dependence to the $4 \mathrm{~d}^{-1}$ widths in $\mathrm{XeF}_{2}$ at a photon energy of $94 \mathrm{eV}$. They also presented a model based on the character of the relevant molecular orbitals that rationalized which states had the greatest width. However, their widths for the corresponding $4 \mathrm{~d}^{-1}$ states of atomic Xe were approximately a factor of two larger than those determined in more recent studies.

The present substate-dependent widths extracted from the $\mathrm{Xe}$ and $\mathrm{XeF}_{2} 4 \mathrm{~d}^{-1}$ photoelectron spectra are shown in Figure S3 of the Supplemental Material for photon energies between 75 and $250 \mathrm{eV}$. The spectra at lower photon energies are asymmetrically broadened by post-collision interactions, and the present analysis is thus limited to higher energies. Two observations can be made directly from the data. First, as observed by Cutler et al., ${ }^{36}$ the widths for $\mathrm{XeF}_{2}$ are systematically larger than those for atomic Xe. This observation has been used by Buth et al. ${ }^{39}$ as evidence for the contribution of interatomic Coulombic decay (ICD) to the Auger decay mechanism in the xenon fluorides. Second, the peak widths are essentially constant as a function of the photon energy. We also observed constant widths for Xe, which is consistent with the results of Juvensuu et al., ${ }^{22}$ although Ausmees et al. ${ }^{21}$ have previously found a photon energy dependence to the $4 \mathrm{~d}^{-1}$ widths for Xe.

Table I shows the substate-dependent widths obtained by averaging the values determined for photon energies between 140 and $250 \mathrm{eV}$. As in the case of the Xe data, the present values for the $\mathrm{XeF}_{2}$ substates are approximately a factor of two smaller than those of Cutler et al. ${ }^{36}$ However, their discussion of the relative widths of the different $\mathrm{XeF}_{2}$ substates is consistent with 
our observations. In particular, the one-electron orbitals associated with the $4 \mathrm{~d}_{5 / 2}{ }^{-1}{ }^{2} \Sigma_{1 / 2}{ }^{+}$and $4 \mathrm{~d}_{3 / 2}{ }^{-1} \prod_{1 / 2}$ substates have the greatest amount of $d_{z^{2}}$ character (i.e., along the F-Xe-F axis), and these substates also have the largest widths.

\section{Auger Processes}

The spectra recorded with $\theta=0^{\circ}$ at 90,120 , and $150 \mathrm{eV}$ in connection with the shake-up processes also include the Auger spectra for $\mathrm{XeF}_{2}$. Figure 10 shows the spectrum recorded at 90 $\mathrm{eV}$, in which the Auger electrons are best isolated from the other photoelectron bands. The

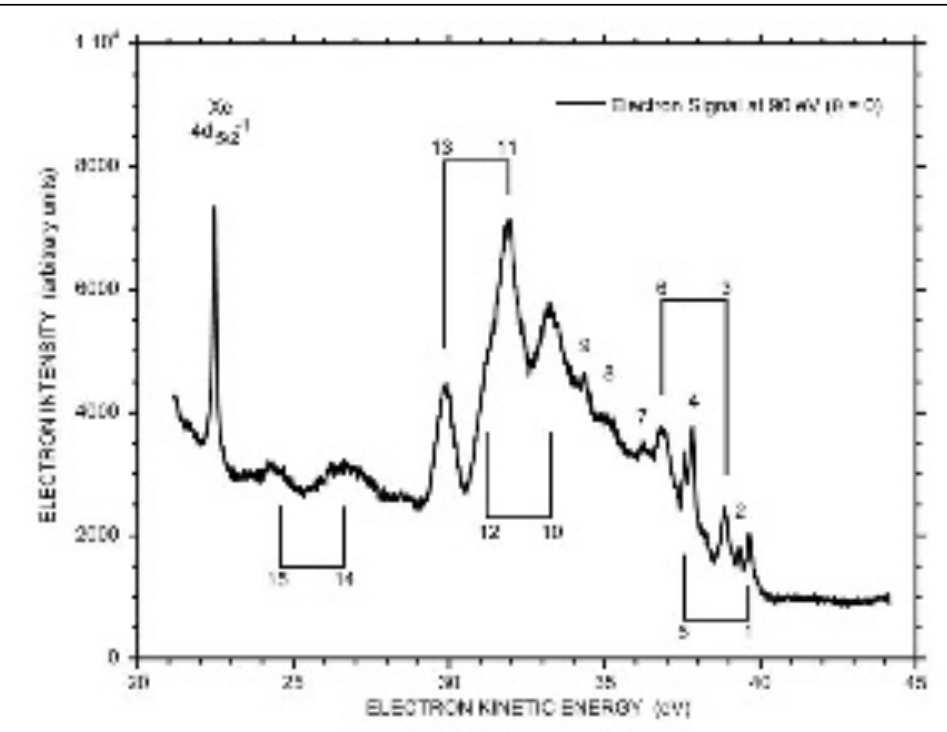

Figure 10. The Auger-electron spectrum recorded at 90 eV photon energy and $\theta=0^{\circ}$. The sharp peak at 22.459 $\mathrm{eV}$ is from the $4 \mathrm{~d}_{5 / 2}{ }^{-1}$ photoionization of atomic Xe. The lines connect pairs of peaks separated by the spin-orbit splitting in $\mathrm{XeF}_{2}\left(4 \mathrm{~d}^{-1}\right)$, suggesting that they result from decay into the same final state. double ionization threshold for $\mathrm{XeF}_{2}$ is expected to be $\sim 30 \mathrm{eV}$, and the highest energy ligandfield- and spin-orbit-split $4 \mathrm{~d}^{-1}$ level is at $72.538 \mathrm{eV}$, which means that the highest energy Auger electron will have a kinetic energy of $\sim 40 \mathrm{eV}$. This expectation is consistent with the spectrum in Figure 10. Note that the sharp peak at $22.459 \mathrm{eV}$ is an impurity line from atomic $\mathrm{Xe}$ $4 \mathrm{~d}_{5 / 2^{-1}}$ photoionization. Although

the $\mathrm{Xe}$ concentration is small, the $\mathrm{XeF}_{2}$ Auger intensities are also small, which makes the $\mathrm{Xe}$ photoelectron peak look relatively intense by comparison.

The Auger spectrum following $4 \mathrm{~d}$ ionization of atomic Xe is split into two groups of lines. ${ }^{24,79}$ The first lies at kinetic energies between 29.9 and $36.5 \mathrm{eV}$, and corresponds to $\mathrm{Xe}^{2+}$ final states with the $\ldots .5 \mathrm{~s}^{2} 5 \mathrm{p}^{4}$ configuration, while the second lies at energies between 19.1 and $24.1 \mathrm{eV}$, and 
corresponds to final states with the $\ldots .5 \mathrm{~s}^{1} 5 \mathrm{p}^{5}$ configuration. The corresponding $\mathrm{XeF}_{2} 4 \mathrm{~d}^{-1}$ Auger spectrum spans a somewhat larger range of kinetic energies, and the splitting between the two regions is smaller. This observation is consistent with the much broader range in energies of the $\mathrm{XeF}_{2}{ }^{+}$and $\mathrm{XeF}_{2}{ }^{2+}$ outer-valence states, as compared to $\mathrm{Xe}^{+}$and $\mathrm{Xe}^{2+}$. The $\mathrm{XeF}_{2}$ features at kinetic energies between 30 and $40 \mathrm{eV}$ in Figure 10 most likely correspond to final state configurations with two holes in a combination of the $5 \pi_{\mathrm{u}}, 10 \sigma_{\mathrm{g}}, 3 \pi_{\mathrm{g}}$, and $4 \pi_{\mathrm{u}}$ orbitals ("outerouter" states in the language of $\AA$ gren ${ }^{80}$ ), while the higher binding energy (lower kinetic energy) features may involve configurations with holes in the $6 \sigma_{\mathrm{u}}$ (predominantly Xe $5 \mathrm{~s}$ character) orbitals as well ("outer-inner" states).

As discussed in detail by Buth et al., ${ }^{38,39}$ however, the whole molecular orbital picture becomes suspect for the doubly ionized states of $\mathrm{XeF}_{2}$ as a result of configuration mixing among the twohole states and three-hole, one-particle states. In addition, the two-hole configurations give rise to a very large number of states very close to the double ionization threshold. For example, Buth et al.$^{39}$ found 38 electronic states of $\mathrm{XeF}_{2}{ }^{2+}$ within $10 \mathrm{eV}$ of the double ionization threshold, and 19 states in the first $5 \mathrm{eV}$. Thus, the prospects for a detailed assignment without additional theoretical input is not promising. In spite of this situation, the Auger spectrum between 23 and $40 \mathrm{eV}$ is actually relatively simple, with only a handful of well-defined features. This observation suggests that there are simplifying aspects to the Auger decay that may ultimately allow more definitive assignments.

On closer examination, the highest energy Auger line in Figure 10 occurs at a kinetic energy of $39.71 \mathrm{eV}$. Assuming this results from the decay of the $\mathrm{XeF}_{2} 4 \mathrm{~d}_{3 / 2}{ }^{-1} \prod_{1 / 2}$ state at $72.538 \mathrm{eV}$, this kinetic energy corresponds to a double ionization threshold of $32.83 \mathrm{eV}$. This value is only slightly lower than the double ionization threshold for atomic Xe $(33.105 \pm 0.004 \mathrm{eV}),{ }^{24}$ and somewhat higher than the lowest $\mathrm{XeF}_{2}$ double ionization potential calculated by Buth et al. $(30.511 \mathrm{eV}),{ }^{39}$ and in reasonably good agreement with the value of Haiduke et al. $(32.3 \mathrm{eV}) .{ }^{41}$ 
The results of Buth et al. indicate that the ground state of $\mathrm{XeF}_{2}{ }^{2+}$ can be reasonably welldescribed as a ${ }^{3} \Sigma_{\mathrm{g}}-$ state arising from two holes in the $5 \pi_{\mathrm{u}}$ HOMO of the neutral molecule. Note, however, that they calculate two additional $5 \pi_{\mathrm{u}}^{-2}$ hole states with ${ }^{1} \Delta_{\mathrm{g}}$ and ${ }^{1} \Sigma_{\mathrm{g}}{ }^{+}$symmetry within $0.3 \mathrm{eV}$ of the ${ }^{3} \sum_{\mathrm{g}}{ }^{-}$state.

If the highest energy Auger peak corresponds to decay from $4 \mathrm{~d}_{3 / 2}{ }^{-1} \prod_{1 / 2}$ into the ground state of $\mathrm{XeF}_{2}^{2+}$, the highest energy Auger peak from a $4 \mathrm{~d}_{5 / 2}{ }^{-1}$ state will be for the analogous process from the ${ }^{2} \Sigma_{1 / 2}$ state, with a kinetic energy of $37.75 \mathrm{eV}$. Thus, all of the peaks above this kinetic energy must arise from one of the two $4 \mathrm{~d}_{3 / 2^{-1}}$ states. Unfortunately, even with this limitation, the number of possible final states precludes a definitive assignment. A number of factors add to the difficulty of making detailed assignments for the remainder of the Auger spectrum. First, propensity rules favoring the production of singlet states of the dications of lighter molecules ${ }^{80,81}$ are likely to break down for a heavy molecule like $\mathrm{XeF}_{2}$. Similarly, while Auger intensities often display site specificity, Buth et al. ${ }^{36}$ have shown that ICD processes contribute approximately $33 \%$ of the total width in the $4 \mathrm{~d}^{-1}$ Auger decay of $\mathrm{XeF}_{2}$, which makes such arguments somewhat suspect. Finally, although the $4 \mathrm{~d}$ electrons in $\mathrm{XeF}_{2}$ are primarily non-bonding, and there is little evidence for vibrational excitation in the singly charged cation, the loss of two valence electrons is more likely to affect the bonding in the doubly charged ion, and thus vibrational excitation is expected to create additional complexity in the Auger electron spectrum.

While not resolution limited, the widths of the $4 \mathrm{~d}_{3 / 2}{ }^{-1}$ peaks with kinetic energies greater than $37.75 \mathrm{eV}$ are considerably sharper than those with lower kinetic energies (i.e., to those $\mathrm{XeF}_{2}{ }^{2+}$ states with higher internal energy). As discussed in the next section, this does not appear to be a simple case of the lines broadening as a result of an increased number of overlapping transitions. Rather, this observation suggests that a faster decay channel for the $\mathrm{XeF}_{2}^{2+}$ sets in about $2 \mathrm{eV}$ above its threshold of formation. Characterization of the ion fragmentation patterns in coincidence with the Auger electrons should be revealing in this regard. 
At kinetic energies below $\sim 37.75 \mathrm{eV}$, the Auger peaks are relatively broad, and the ligand-fieldsplit states are not likely to be resolved. However, for Auger processes from the $\mathrm{XeF}_{2} 4 \mathrm{~d}_{5 / 2}{ }^{-1}$ and $4 \mathrm{~d}_{3 / 2}{ }^{-1}$ spin-orbit states that access the same final states, pairs of Auger peaks should be observed that are separated by approximately the ligand-field state-averaged spin-orbit splitting of $\sim 2.01$ $\mathrm{eV}$. Several such pairs of peaks are clearly observed in Figure 10. Table IV gives the energies of the numbered peaks, along with tentative assignments for the spin-orbit symmetry of the initial state in the Auger decay for the paired transitions.

\section{E. Resonant Auger Processes}

Resonant Auger spectra of $\mathrm{XeF}_{2}$ were recorded at 61.526, 63.455, and $67.596 \mathrm{eV}$, corresponding to the $\left(\mathrm{Xe} 4 \mathrm{~d}_{5 / 2^{-1}}\right) \sigma^{*},\left(\mathrm{Xe} 4 \mathrm{~d}_{3 / 2^{-1}}\right) \sigma^{*}$, and $\left(\mathrm{Xe}_{4} \mathrm{~d}_{5 / 2^{-1}}\right) 6 \mathrm{p} \pi$ resonances, respectively, as well as at two off-resonance energies of 59.50 and $62.60 \mathrm{eV}$. Spectra were recorded at both $\theta=0^{\circ}$ and $90^{\circ}$, allowing the determination of the corresponding $\beta$ parameters. Figure 11 shows the resonant

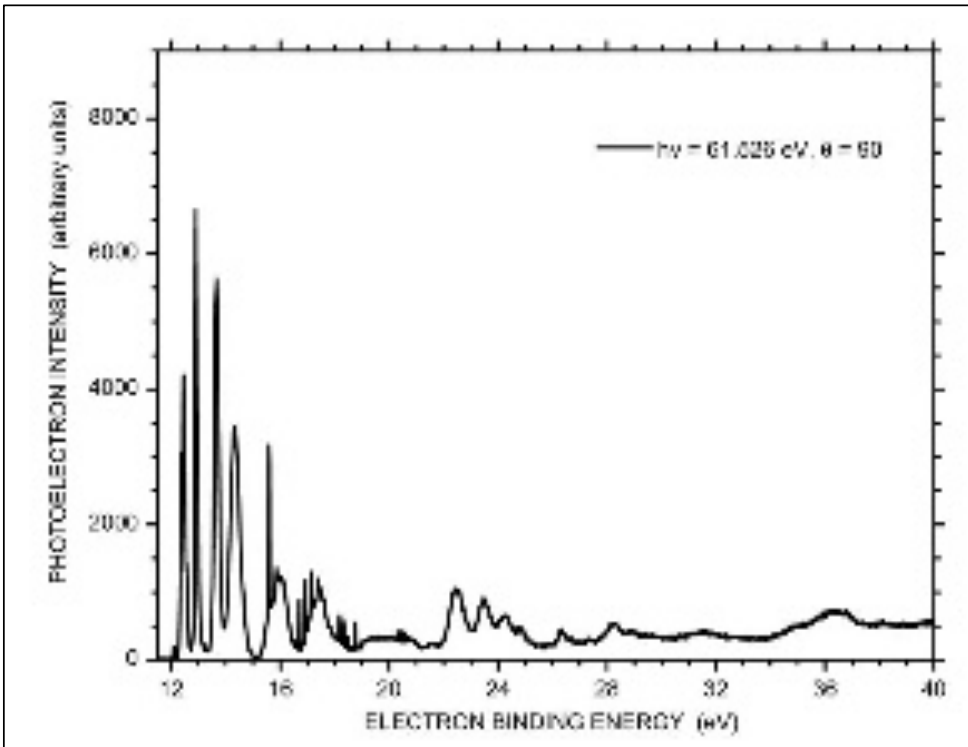

Figure 11. The resonant Auger spectrum recorded at the $\left(\mathrm{Xe} 5 / 2^{-1}\right) \sigma^{*}$ resonance of $\mathrm{XeF}_{2}$ at $61.526 \mathrm{eV}$ and plotted vs. the electron binding energy. The assignments of the impurity bands are indicated in the Supplemental Material.
Auger spectrum recorded at the $\left(\mathrm{Xe} 4 \mathrm{~d} 5 / 2^{-1}\right) \sigma^{*}$ resonance with $\theta$ $=90^{\circ}$, and plotted vs. electron binding energy. An expanded version of the region between threshold and $30 \mathrm{eV}$ is shown in the Supplemental Material, along with the identification of a number of impurity bands due to $\mathrm{N}_{2},{ }^{82}$ and $\mathrm{O}_{2} \cdot{ }^{83}$ These impurity bands were caused by a very small air leak in the inlet system. 
While somewhat of a nuisance, these bands allow the accurate calibration of the spectrum and allow the matching of intensities for background subtraction. "Magic-angle" photoelectron spectra and the corresponding $\beta$ values for the three resonance positions are also provided in the Supplemental Material.

At binding energies below $\sim 16.5 \mathrm{eV}$, all of the spectra look quite similar, displaying the $5 \pi_{\mathrm{u}}{ }^{-1}$, $10 \sigma_{\mathrm{g}}{ }^{-1}, 3 \pi_{\mathrm{g}}{ }^{-1}$, and $4 \pi_{\mathrm{u}}{ }^{-1}$ valence bands resulting from single-photon ionization of the ground state molecule. ${ }^{84}$ Magic angle spectra were synthesized using Equation 6, and these were normalized to each other by using the intensities of the impurity bands. The resulting $\mathrm{XeF}_{2}$ band intensities on resonance are within $10-15 \%$ of the intensities off resonance, suggesting that the participator Auger processes are relatively weak. This result is consistent with the resonant Auger spectrum of atomic Xe. In particular, the atomic Xe 4d $5 / 26 \mathrm{p}$ lifetime width is $0.1098 \pm 0.0010 \mathrm{eV},{ }^{20}$ which

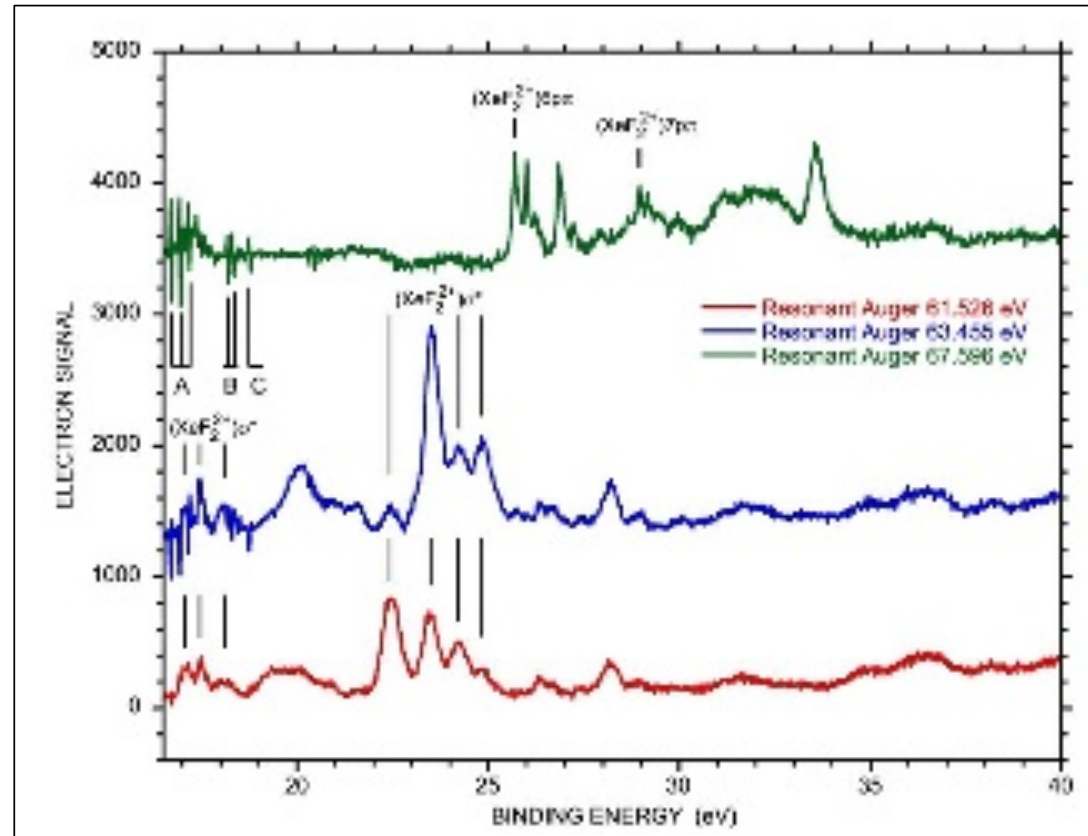

Figure 12. The difference photoelectron spectra recorded at $90^{\circ}$ in which the off-resonance spectrum recorded at $62.50 \mathrm{eV}$ was subtracted from the spectra for the $\left(4 \mathrm{~d}_{5 / 2^{-1}}\right) \sigma^{*},\left(4 \mathrm{~d}_{3 / 2^{-1}}\right) \sigma^{*}$, and $\left(4 \mathrm{~d}_{5 / 2^{-1}}\right) 6 \mathrm{p} \pi$ resonances. See text for details. The sharp spikes labeled A, B, and C correspond to imperfectly subtracted impurity bands corresponding to vibrational levels of the $\mathrm{N}_{2}{ }^{+}$ $\mathrm{A}^{2} \Pi_{\mathrm{u}}, \mathrm{O}_{2}{ }^{+} \mathrm{b}^{4} \Sigma_{\mathrm{g}}$, and $\mathrm{N}_{2}{ }^{+} \mathrm{B}{ }^{2} \Sigma_{\mathrm{u}}{ }^{+}$states, respectively. is very close to the $\mathrm{Xe} 4 \mathrm{~d}_{5 / 2^{-1}}$ Auger lifetime of $0.111 \pm$ $0.003 \mathrm{eV} .^{22}$ This observation suggests that spectator Auger processes will dominate the decay following resonant $\left(4 d^{-1}\right) 6 p$ excitation in $\mathrm{Xe}$, as is generally found. This result also suggests that spectator decay will dominate in the decay of the $\left(4 \mathrm{~d}_{5 / 2^{-1}}\right) 6 \mathrm{p} \pi$ resonance in $\mathrm{XeF}_{2}$ as well. The situation could be somewhat different for the 
lower-lying $\left(4 \mathrm{~d}^{-1}\right) \sigma^{*}$ resonances in $\mathrm{XeF}_{2}$, but the data below clearly show that spectator decay is the stronger process for these as well.

Above $16.5 \mathrm{eV}$, a number of new bands are observed in the resonant spectra, along with the usual $6 \sigma \mathrm{u}^{-1}$ and $9 \sigma_{\mathrm{g}}{ }^{-1}$ valence bands at 17.39 and $26.28 \mathrm{eV}$, respectively. ${ }^{33}$ To enhance the features in the resonant spectra, we have subtracted the corresponding nonresonant spectrum at $62.50 \mathrm{eV}$ from each of the resonant spectra at $\theta=0^{\circ}$ and $90^{\circ}$. Figure 12 shows the resulting $90^{\circ}$ difference spectra for the three resonances over the energy range from 16.5 to $40 \mathrm{eV}$. The signal at the lowest kinetic energies is somewhat noisy because it is overlapped by both the $6 \sigma_{u}{ }^{-1}$ band and $\mathrm{N}_{2}$ and $\mathrm{O}_{2}$ impurity bands, and the subtraction is imperfect.

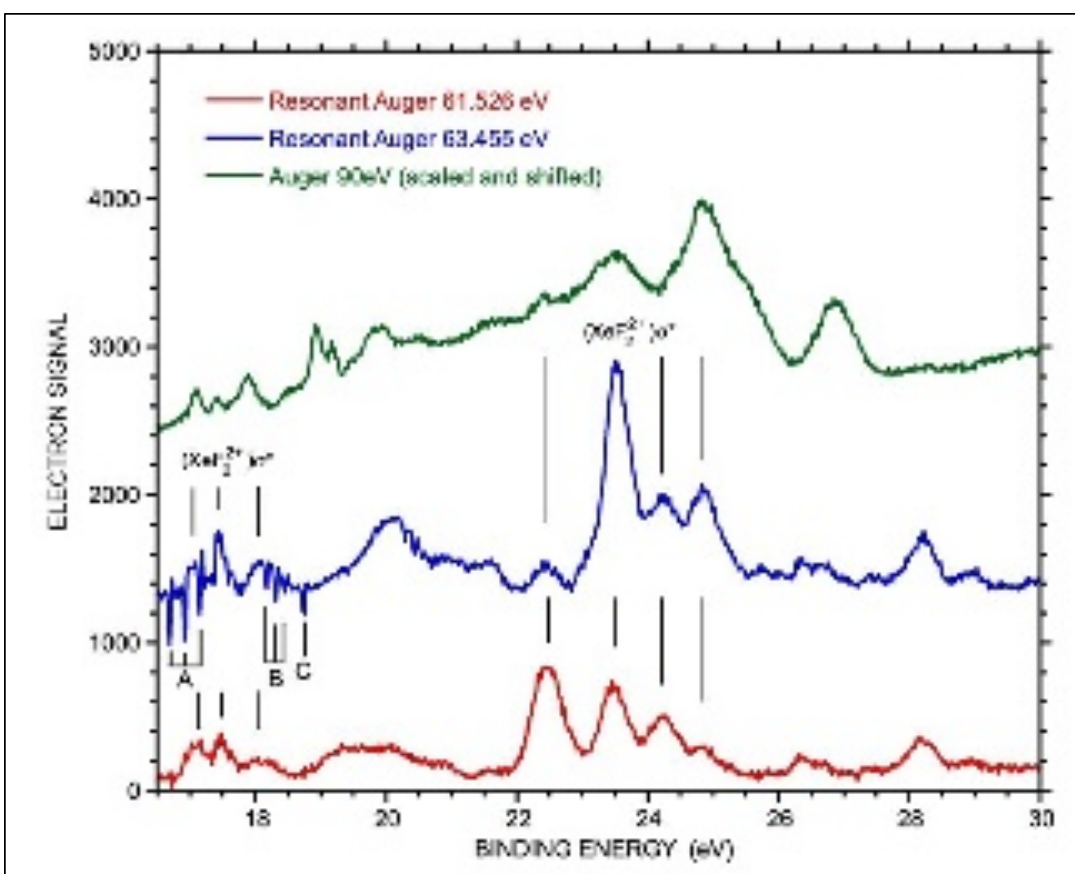

Figure 13. Expanded portions of the $\left(4 \mathrm{~d}_{5 / 2^{-1}}\right) \sigma^{*}$ and $\left(4 \mathrm{~d}_{3 / 2^{-1}}\right) \sigma^{*}$ spectra from Figure 12, along with the normal $\theta=0^{\circ}$ Auger electron spectrum recorded at $90 \mathrm{eV}$, where the $\mathrm{x}$-axis of the latter has been reversed and shifted to line up with the lowest binding energy feature of the $\sigma^{*}$ spectra. The sharp spikes labeled A, B, and C correspond to imperfectly subtracted impurity bands corresponding to vibrational levels of the $\mathrm{N}_{2}{ }^{+}$ $\mathrm{A}^{2} \prod_{\mathrm{u}}, \mathrm{O}_{2}{ }^{+} \mathrm{b}^{4} \Sigma_{\mathrm{g}}$, and $\mathrm{N}_{2}{ }^{+} \mathrm{B}^{2} \Sigma_{\mathrm{u}^{+}}$states, respectively.
The resonant Auger spectra for the $\mathrm{XeF}_{2}\left(4 \mathrm{~d}_{5 / 2^{-1}}\right) \sigma^{*}$ and $\left(4 \mathrm{~d}_{3 / 2^{-1}}\right) \sigma^{*}$ resonances show most of the same features between 16.5 and $40 \mathrm{eV}$, although the relative intensities vary considerably. In contrast, the spectrum recorded on the $\left(4 \mathrm{~d} 5 / 2^{-1}\right) 6 \mathrm{p} \pi$ resonance is quite different. We focus first on the decay of the two $\sigma^{*}$ resonances, and then contrast this with a discussion of the $6 \mathrm{p} \pi$ resonance. 
The spectator Auger spectra are expected to show strong similarities with the true Auger spectrum. However, because the final state in resonant Auger decay is a singly charged cation rather than a double charged cation, and because the excited electron is somewhat effective in screening the nuclear charge, the kinetic energies of the peaks are shifted to considerably higher energy that the corresponding peaks in the non-resonant Auger spectrum. The excited electron can also lead to splitting in the Auger peaks depending on the symmetry of the excited orbital, but for the $\sigma^{*}$ orbital it is only the spin-splitting, which is expected to be small.

The first few peaks at binding energies of 16.5 to $18.5 \mathrm{eV}$ in both the $\left(4 \mathrm{~d}_{5 / 2}{ }^{-1}\right) \sigma^{*}$ and $\left(4 \mathrm{~d}_{3 / 2^{-1}}\right) \sigma^{*}$ resonant Auger spectra bear a striking resemblance to the highest energy features in the true Auger spectrum of Figure 10. Figure 13 shows an expanded version of the two $\sigma^{*}$ spectra, as well as the Auger spectrum, in which the x-axis of the latter has been reversed and shifted to line up with the lowest binding energy feature in the $\sigma^{*}$ spectra. Note that the Auger spectrum contains contributions from the decay of both the $4 \mathrm{~d}_{5 / 2}{ }^{-1}$ and $4 \mathrm{~d}_{3 / 2}{ }^{-1}$ states of $\mathrm{XeF}_{2}{ }^{+}$, and by plotting in this manner, only the $4 \mathrm{~d}_{3 / 2}{ }^{-1}$ features are expected to line up with the $\sigma^{*}$ features. Nevertheless, the overall shapes of the resonant and true Auger spectra appear quite similar. The binding energy with respect to the double ionization threshold of the lowest energy feature in the $\sigma^{*}$ spectra is quite substantial. In particular, assuming the lowest energy spectator Auger feature at $17.09 \mathrm{eV}$ is based on the lowest electronic state of $\mathrm{XeF}_{2}{ }^{2+}$, the binding energy is $15.74 \mathrm{eV}$. This large value reflects the low effective principal quantum number of the $\sigma^{*}$ electron (it is effectively a $5 p$ electron).

The similarity between the resonant Auger spectra for the $\left(4 \mathrm{~d}_{5} / 2^{-1}\right) \sigma^{*}$ and $\left(4 \mathrm{~d}_{3 / 2}{ }^{-1}\right) \sigma^{*}$ resonances and the regular Auger spectrum extends to the linewidths of the lowest few features in the resonant Auger spectra. While these features are quite sharp, from just $2 \mathrm{eV}$ above the lowest feature the linewidth appears to increase substantially. Unlike the regular Auger spectra, the resonant Auger spectra are state selected, and the increased linewidth does not appear to be 
simply due to an increased density of transitions. This observation suggests that the $\mathrm{XeF}_{2}{ }^{2+}$ ion core of the $\left(\mathrm{XeF}_{2}^{2+}\right) \sigma^{*}$ states populated in the resonant Auger decay has similar dissociation/decay dynamics as the free $\mathrm{XeF}_{2}{ }^{2+}$ ion.

The similarity of the $\left(4 \mathrm{~d}_{5} / 2^{-1}\right) \sigma^{*}$ and $\left(4 \mathrm{~d}_{3} / 2^{-1}\right) \sigma^{*}$ spectra between 16.5 and $30 \mathrm{eV}$ suggests that the Auger decay of the corresponding cores access the same final states, albeit with different relative intensities. This behavior is extended to the higher-lying states as well. Here, the correspondence with the true Auger spectrum is somewhat less obvious, but a clear similarity remains.

Interestingly, some of the higher energy features are significantly stronger than the lowest energy features, suggesting that decay to molecular ion states based on the $\mathrm{Xe}^{2+}{ }^{1} \mathrm{D}_{2}$ and ${ }^{1} \mathrm{~S}_{0}$ states is enhanced relative to those based on the ${ }^{3} \mathrm{P}_{\mathrm{J}}$ states. As discussed for the Auger spectra, this interpretation is complicated by the large number of low-lying states of the doubly ionized molecule. ${ }^{39}$

The $67.596 \mathrm{eV}$ spectrum of $\mathrm{XeF}_{2}$ corresponds to excitation of the $\left(4 \mathrm{~d}_{5 / 2}{ }^{-1}\right) 6 \mathrm{p} \pi$ Rydberg state, and spectator Auger decay is expected to populate the $6 \mathrm{p} \pi$ Rydberg states converging to low-lying electronic states of $\mathrm{XeF}_{2}{ }^{+}$. For atomic Xe, the lowest lying $\mathrm{Xe}^{2+}\left({ }^{3} \mathrm{P}_{2}\right) 6 \mathrm{p}$ Rydberg state is bound by $7.114 \mathrm{eV}$ relative to the $\mathrm{Xe}^{2+}{ }^{3} \mathrm{P}_{2}$ ground state $\left(33.3397 \mathrm{eV}\right.$ above $\left.\mathrm{Xe}^{1} \mathrm{~S}_{0}\right)$. The lowest-lying feature in the $\mathrm{XeF}_{2}\left(4 \mathrm{~d}_{5 / 2^{-1}}\right) 6 \mathrm{p} \pi$ resonant Auger spectrum lies at $25.70 \mathrm{eV}$, or $7.13 \mathrm{eV}$ below the $\mathrm{XeF}_{2}{ }^{2+}$ threshold, clearly suggesting a spectator process to a $6 \mathrm{p} \pi$ state of the singly charged cation. The structure of the lowest lying features in the $6 \mathrm{p} \pi$ spectrum show somewhat different splittings than the $\sigma^{*}$ spectra, reflecting the coupling of the $6 \mathrm{p} \pi$ electron with the different ioncore states. Most of the remaining structure in the $6 \mathrm{p} \pi$ spectrum has splittings of 0.2 to $0.4 \mathrm{eV}$, making it unlikely to be vibrational structure, and more likely reflects decay to different $\mathrm{XeF}_{2}{ }^{2+}$ states. The features between 25.5 and $27.5 \mathrm{eV}$ are also relatively sharp, which is consistent with the longer lifetimes of the corresponding $\left(4 \mathrm{~d}^{-1}\right) \sigma^{*}$ states and the relative stability of these $\left(\mathrm{XeF}_{2}^{2+}\right) 6 \mathrm{p} \pi$ states. 
Shake-up processes are often significant in resonant Auger spectra. The lowest $\left(\mathrm{Xe}^{2+}\right) 7 \mathrm{p}$ state has a binding energy of $3.725 \mathrm{eV},{ }^{85}$ while the $\mathrm{XeF}_{2}$ feature at $28.957 \mathrm{eV}$ has a binding energy of 3.87 $\mathrm{eV}$. This observation strongly suggests an assignment to the lowest $\left(\mathrm{XeF}_{2}{ }^{2+}\right) 7 \mathrm{p} \pi$ Rydberg state, and neighboring features probably reflect additional core states. Not unexpectedly, the two $\sigma^{*}$ resonant Auger spectra do not show significant features that match up with the $6 \mathrm{p} \pi$ and $7 \mathrm{p} \pi$ features in the $\left(4 \mathrm{~d}_{5 / 2^{-1}}\right) 6 \mathrm{p} \pi$ spectrum. This result is consistent with the shake-up process preserving the 1 symmetry of the excited electron. On the other hand, a stronger feature is observed in the $\sigma^{*}$ spectra at $\sim 26.32 \mathrm{eV}$, or $0.62 \mathrm{eV}$ above the $6 \mathrm{p} \pi$ in the $\left(4 \mathrm{~d}_{5 / 2}{ }^{-1}\right) 6 \mathrm{p} \pi$ spectrum. This feature is likely a result of shake-up from the $\sigma^{*}$ into the $6 \mathrm{p} \sigma$ orbital. Interestingly, Comes et al. assign the $\left(4 \mathrm{~d}_{5} / 2^{-1}\right) 6 \mathrm{p} \sigma$ transition to a feature lying $\sim 0.78 \mathrm{eV}$ above the $\left(4 \mathrm{~d}_{5 / 2}{ }^{-1}\right) 6 \mathrm{p} \pi$ transition, in reasonably good agreement with the present splitting observed in the lower-lying states.

\section{CONCLUSIONS}

We have presented an extensive experimental study of the $4 \mathrm{~d}^{-1}$ ionization of $\mathrm{XeF}_{2}$, including ionyield curves, and ligand-field- and spin-orbit-resolved photoelectron angular distributions and branching fractions. The overall behavior closely follows the corresponding ion yields, angular distributions, and branching fractions for atomic Xe, and some of the results are reproduced reasonably well by theoretical calculations of the photoionization process. In particular, although the theoretical $\mathrm{XeF}_{2}$ cross section between the $4 \mathrm{~d}$ threshold and the $250 \mathrm{eV}$ does not agree well with experiment, the corresponding $\beta$ values and branching fractions are fairly well reproduced. Ligand-field - and spin-orbit-resolved lifetime widths were also measured for the resulting photoions, and these are consistent with the widths observed for atomic Xe. We also discussed the shake-up processes accompanying photoionization and the Auger-electron spectra resulting from the decay of the photoion. Finally, resonant Auger spectra were recorded just below the $4 \mathrm{~d}$ threshold for the $\left(4 \mathrm{~d}_{5 / 2}\right) \sigma^{*},\left(4 \mathrm{~d}_{3 / 2}\right) \sigma^{*}$, and $\left(4 \mathrm{~d}_{5 / 2}\right) 6 \mathrm{p} \pi$ resonances. These spectra show many 
features in common with the normal Auger spectra. Interestingly, both the Auger and resonant Auger spectra appear to be relatively simple, in spite of the large number of doubly ionized $\mathrm{XeF}_{2}$ states close to threshold. Understanding the source of this simplicity is an interesting problem for the future.

The present results suggest a number of future experiments. As shown by Southworth et al. at the $3 \mathrm{~d}$ edge of $\mathrm{XeF}_{2},{ }^{35}$ neutral and ionic fragmentation patterns can provide a different window into the competing decay processes of the primary photoions or resonant levels of the neutral. Second, coincidence measurements of the photoelectron and Auger electron such as those performed on atomic Xe would help to disentangle the Auger spectrum. ${ }^{15,27,28,74}$ Finally, comprehensive resonant Auger measurements of a larger set of pre-threshold resonances could provide considerable insight into the Rydberg states of the singly charged cation converging to the doubly charged ion, as well as into the associated shake-up and shake-down processes. Such comprehensive measurements on prototypical heavy atom systems like $\mathrm{XeF}_{2}$ will also help provide a framework for a more detailed analysis of novel time-resolved x-ray studies, such as the recent two-color pump-probe experiments on $\mathrm{XeF}_{2}$ performed using a free-electron $\mathrm{x}$-ray laser source. ${ }^{86}$

\section{SUPPLEMENTAL MATERIAL}

The Supplemental Material contains a schematic figure of the interaction region, along with additional figures of the energy-dependent Auger widths, and expanded plots of the resonant Auger spectra and magic-angle Auger spectra.

\section{ACKNOWLEDGEMENTS}

We would like to thank C. Buth and L. Cederbaum for making the digital data from their calculations on the double ionization potentials of $\mathrm{XeF}_{2}$ available to us, and for helping us with their interpretation. D.M.P.H. is grateful to the Science and Technology Facilities Council 
(United Kingdom) for financial support. S.T.P. is supported by the U.S. Department of Energy, Office of Science, Office of Basic Energy Sciences, Division of Chemical Sciences,

Geosciences, and Biosciences under contract No. DE-AC02-06CH11357. We are grateful to the SOLEIL staff for running the facility and providing beamtime under Project No. 20190046.

\section{DATA AVAILABILITY STATEMENT}

The data that support the findings of this study are available from the corresponding author upon reasonable request.

\section{AUTHOR DECLARATIONS}

The authors have no conflicts of interest to disclose. 


\section{REFERENCES}

1. K. Codling and R. P. Madden, Phys. Rev. Lett. 12, 106 (1964).

2. A. Starace, Phys. Rev. A 2, 118 (1970).

3. D. J. Kennedy and S. T. Manson, Phys. Rev. A 5, 227 (1972).

4. S. P. Shannon, K. Codling, and J. B. West, J. Phys. B 10, 825 (1977).

5. G. C. King, M. Tronc, F. H. Read, and R. C. Bradford, J. Phys. B 10, 2479 (1977).

6. J. B. West and J. Morton, At. Data Nucl. Data Tables 22, 103 (1978).

7. K. T. Cheng and W. R. Johnson, Phys. Rev. A 28, 2820 (1983).

8. D. J. Bristow, J. S. Tse, and G. M. Bancroft, Phys. Rev. A 25, 1 (1982).

9. S. Southworth, U. Becker, C. M. Truesdale, P. H. Kobrin, D. W. Lindle, S. Owaki, and D. A. Shirley, Phys. Rev. A 28, 261 (1983).

10. D. W. Lindle, T. A. Ferrett, P. A. Heimann, and D. A. Shirley, Phys. Rev. A 37, 3808 (1988).

11. S. Svensson, B. Eriksson, N. Mårtensson, G. Wendin, and U. Gelius, J. Electron Spectrosc. Relat. Phenom. 47, 327 (1988).

12. B. Kämmerling, H. Kossman, and V. Schmidt, J. Phys. B 22, 841 (1989).

13. U. Becker, D. Szostak, H. G. Kerkhoff, M. Kupsch, B. Langer R. Wehlitz, A. Yagashita, and T. Hayaishi, Phys. Rev. A 39, 3902 (1989).

14. N. Shanthi and P. C. Deshmukh, Phys. Rev. A 40, 2400 (1989).

15. B. Kämmerling, B. Krässig, and V. Schmidt, J. Phys. B 23, 4487 (1990).

16. T. Hayaishi, E. Murakami, Y. Morioka, H. Aksela, S. Aksela, E. Shigemasa, and A. Yagashita, Phys. Rev. A 44, R2771 (1991).

17. W. F. Chan. G. Cooper, X. Guo, G. R. Burton, and C. E. Brion, Phys. Rev. A 46, 149 (1992).

18. A. Ausmees, S. J. Osborne, R. Moberg, S. Svensson, S. Aksela, O.-P Sairanen, A. Kivimäki, A. Naves de Brito, E. Nõmmiste, J. Jaihiainen, and H. Aksela, Phys. Rev. A 51, 855 (1995). 
19. A. Ausmees, S. J. Osborne, S. Svensson, A. Naves de Brito, O.-P Sairanen, A. Kivimäki, E. Nõmmiste, H. Aksela, and S. Aksela, Phys. Rev. A 52, 2943 (1995).

20. O.-P Sairanen, A. Kivimäki, E. Nõmmiste, H. Aksela, and S. Aksela, Phys. Rev. A 54, 2834 (1995).

21. A. Ausmees, A. Hahlin, S. L. Sorensen, S. Sundin, I. Hjelte, O. Björneholm, and S. Svensson, J. Phys. B 32, L197 (1999).

22. M. Jurvansuu, A. Kivimäki, and S. Aksela, Phys. Rev. A 64, 012502 (2001).

23. H. Wang, G. Snell, O. Hemmers M. M. Sant'Anna, I. Sellin. N. Berrah. D. W. Lindle, P. C. Deshmukh. N. Haque, and S. T. Manson, Phys. Rev. Lett. 87, 123004 (2001).

24. T. X. Carroll, J. D. Bozek, E. Kukk, V. Myrseth, L. J. Saethre, T. D. Thomas, and K. Weisner, J. Electron Spectrosc. Rel. Phenom. 125, 127 (2002).

25. F. Penent, J. Palaudoux, P. Lablanquie, L. Andric, R. Feifel, and J. H. D. Eland, Phys. Rev. Lett. 95, 083002 (2005).

26. It has been recently proposed to change the name "Auger decay" to "Auger-Meitner decay" to recognize the work of Lise Meitner in the discovery of this effect. See: D. Matsakis, A. Coster, B. Laster, and R. Sime, "A renaming proposal: "The Auger-Meitner effect", Phys. Today, 72, 10 (2019).

27. B. Kämmerling, B. Krässig, and V. Schmidt, J. Phys. B. 25, 3621 (1992).

28. B. Kämmerling and V. Schmidt, J. Phys. B 26, 1141 (1993).

29. J. Viefhaus, S. Cvejanović, B. Langer, T. Lischke, G. Prümper, D. Rolles, A. V. Golovin, A. N. Grum-Grzhimailo, N. M. Kabachnik, and U. Becker, Phys. Rev. Lett. 92, 083011 (2004).

30. H. Basch, J. W. Moskowitz, C. Hollister, and D. Hankin, J. Chem. Phys. 55, 1922 (1971).

31. F. J. Comes, R. Haensel, U. Neilsen, and W. H. E. Schwartz, J. Chem. Phys. 58, 516 (1973).

32. T. X. Carroll, R. W. Shaw, T. D. Thomas, C. Kindle, and N. Bartlett, J. Am. Chem. Soc. 96, 1989 (1974).

33. G. M. Bancroft, P. A. Malmquist, S. Svensson, E. Basilier, U. Gelius, and K. Seigbahn, Inorg. Chem. 17, 1595 (1978). 
34. S. Aksela, G. M. Bancroft, D. J. Bristow, H. Aksela, and G. J. Schrobilgen, J. Chem. Phys. 82, 4809 (1985).

35. S. H. Southworth, R. Wehlitz, A. Picón, C. S. Lehmann, L. Cheng, and J. F. Stanton, J. Chem. Phys. 142, 224302 (2015).

36. J. N. Cutler, G. M. Bancroft, J. D. Bozek, K. H. Tan, and G. J. Schrobilgen, J. Am. Chem. Soc. 113, 9125 (1991).

37. G. M. Bancroft, S. Aksela, H. Aksela, K. Gürtler, K. H. Tan, B. W. Yates, and J. S. Tse, J. Phys. B 20, 3057(1987).

38. C. Buth, R. Santra, and L. S. Cederbaum, J. Chem. Phys. 119, 7763 (2003).

39. C. Buth, R. Santra, and L. S. Cederbaum, IJ. Chem. Phys. 119, 10575 (2003).

40. M. Pernpointner and L. S. Cederbaum, J. Chem. Phys. 122, 214302 (2005).

41. R. L. A. Haiduke, H. d. P. M. Filho, and A. B. Ferreira da Silva, Chem. Phys. 348, 89 (2008).

42. R. Forbes, A. De Fanis, C. Bomme, D. Rolles, S. T. Pratt, I. Powis, N. A. Besley, S. Nandi, A. R. Milosavljević, C. Nicolas, J. D. Bozek, J. G. Underwood, and D. M. P. Holland, J. Chem. Phys. 149, 094304 (2018).

43. R. Forbes, A. De Fanis, D. Rolles, S. T. Pratt, I. Powis, N. A. Besley, A. R. Milosavljević, C. Nicolas, J. D. Bozek, and D. M. P. Holland, J. Phys. B 53, 155101 (2020).

44. R. Forbes, A. De Fanis, C. Bomme, D. Rolles, S. T. Pratt, I. Powis, N. A. Besley, M. Simon, S. Nandi, A. R. Milosavljević, C. Nicolas, J. D. Bozek, J. G. Underwood, and D. M. P. Holland, J. Chem. Phys. 149, 144302 (2018).

45. C. N. Yang, Phys. Rev. 74, 764 (1948).

46. G. M. J. Barca, C. Bertoni, L. Carrington, D. Datta, N. De Silva, J. E. Deustua, D. G. Fedorov, J. R. Gour, A. O. Gunina, E. Guidez, T. Harville, S.n Irle, J. Ivanic, K. Kowalski, S. S. Leang, H. Li, W. Li, J. J. Lutz, I. Magoulas, J. Mato, Vladimir Mironov, H. Nakata, B. Q. Pham, P. Piecuch, D. Poole, S. R. Pruitt, A. P. Rendell, L. B. Roskop, K. Ruedenberg, T. Sattasathuchana, M. W. Schmidt, J. Shen, L. Slipchenko, 
M. Sosonkina, V. Sundriyal, A. Tiwari, J. L. Galvez Vallejo, B. Westheimer, M. Włoch, P. Xu, F. Zahariev, andM. S. Gordon. J. Chem. Phys. 152, 154102 (2020).

47. M. W. Schmidt, K. K. Baldridge, J. A. Boatz, S. T. Elbert, Mark S. Gordon, Jan. H. Jensen, S. Koseki, N. Matsunaga, K. A. Nguyen, S. Su, T. L. Windus, M. Dupuis, and J. A. Montgomery. J. Comp. Chem, 141347 (1993).

48. S. Reichman and F. Schreiner. J. Chem. Phys., 51, 2355 (1969).

49. F. A. Gianturco, R. R. Lucchese, and N. Sanna, J. Chem. Phys. 100, 6464 (1994).

50. A. P. P. Natalense and R. R Lucchese. J. Chem. Phys. 111, 5344 (1999).

51. R. R. Lucchese, N. Sanna, A. P. P. Natalense, and F. A. Gianturco, The ePolyScat User's Manual, available at: https://epolyscat.droppages.com/ .

52. J. L. Dehmer, D. Dill, and S. Wallace, Phys. Rev. Lett. 43, 1005 (1979).

53. P. M. Dittman, D. Dill, and J.L. Dehmer, J. Chem. Phys. 76, 5703 (1982).

54. J. R. Swanson, D. Dill, and J.L. Dehmer, J. Phys. B 14, L207 (1981).

55. I. Powis, Phys. Rev. A 84, 013402 (2011).

56. P. Hockett, XeF2 4d photoionization calculations, OSF, 2021, available at: https://doi.org/10.17605/OSF.IO/JWYP7.

57. M. Ya. Amusia and J. P. Connerade, Rep. Prog. Phys. 63, 41 (2000).

58. Y.-J. Chen, S. Pabst, and R. Santra, J. Phys. Commun. 2, 045024 (2018).

59. P. Hockett and K. L. Reid, J. Chem. Phys, 127, 154308, (2007).

60. P. Hockett, "Photoionization Dynamics of Polyatomic Molecules" University of Nottingham. 2009. Available at: http://eprints.nottingham.ac.uk/10857/.

61. Y. Hikosaka, P. Lablanquie, F. Penent, T. Kaneyasu, E. Shigemasa, J. H. D. Eland, T. Aoto, and K. Ito, Phys. Rev. Lett. 98, 183002 (2007).

62. K. Codling and R. P. Madden, Appl. Opt. 4, 1431 (1965).

63. B. W. Yates, K. H. Tan, G. M. Bancroft, L. L. Coatsworth, J. S. Tse, and G. J. Schrobilgen, J. Chem. Phys. 84, 3603 (1986).

64. J. S. Tse, J. Chem. Phys. 89, 928 (1988). 
65. G. B. Armen, J. Tulkki, T. Åberg, and B. Craseman, Phys. Rev. A 36, 5606 (1987).

66. J. A. R. Samson, W. C. Stolte, Z. X. He, J. N. Cutler, and D. Hansen, Phys. Rev. A 54, 2099 (1996).

67. S. Kosugi, M. Iizawa, Y. Kawarai, Y. Kuriyama, A. L. D. Kilcoyne, F. Koike, N. Kuze, D. S. Slaughter, and Y. Azuma, J. Phys. B 48, 115003 (2015).

68. K. N. Huang, W. R. Johnson, and K. T. Cheng, At. Data Nucl. Data Tables 26, 33 (1981).

69. E. Kukk, J. D. Bozek, J. A. Sheehy, P. W. Langhoff, and N. Berrah, J. Phys. B 33, L51 (2000).

70. J. W. Cooper, Phys. Rev. 128, 681 (1962).

71. J. Cooper and R. N. Zare, J. Chem. Phys. 1968, 48, 942; erratum J. Chem. Phys. (1968) 49, 4252.

72. I. Powis, D. M. P. Holland, E. Antonsson, C. Nicolas, C. Miron, M. Schneider, D. Yu. Soshinikov, A. Dreuw, and A. B, Trofimov, J. Chem. Phys 143, 144304 (2015).

73. D. M. P. Holland, S. Nandi C. Niccolas, J. D. Bozek, M. Patanen, and I. Powis, Chem. Phys. 542, 111050 (2021).

74. V. Schmidt, Electron Spectrometry of Atoms using Synchrotron Radiation (Cambridge University Press, Cambridge, UK, 1997).

75. J. S. Tse, D. J. Bristow, G. M. Bancroft, and G. Schrobilgen, Inorg. Chem. 18, 1766 (1979)

76. D. M. P. Holland, private communication (2021).

77. U. Nielsen and W. H. E. Schwarz, Chem. Phys. 13, 195 (1976).

78. N. Saito and I. H. Suzuki, Int. J. Mass Spectrom. Ion Processes, 115, 157 (1992).

79. J. Söderström, A. Lindblad, A. N. Grum-Grzhimailo, O. Travnikova, C. Nicolas, S. Svensson, and C. Miron, New J. Phys. 13, 073014 (2011).

80. H. Ågren, J. Chem. Phys. 75, 1267 (1981).

81. H. Ågren and O. Vahtras, J. Phys. B 26, 913 (1993).

82. P. Baltzer, M. Larsson, L. Karlsson, B. Wannberg, and M. Carlsson Göthe, Phys. Rev. A 46, 5545 (1992). 
83. O. Edqvist, E. Lindholm, L, E. Selin, and L. Åsbrink, Phys. Scripta 1, 25 (1970).

84. C. R. Brundle, M. B. Robin, and G. R. Jones, J. Chem. Phy. 52, 3383-3386 (1970).

85. NIST Atomic Spectra Database at: https://physics.nist.gov/cgi-bin/ASD/levels_hold.pl?el=Xe

86. A. Picon, C. S. Lehmann, C. Bostedt, A. Rudenko, A. Marinelli, T. Osipov, D. Rolles, N. Berrah, C. Bomme, M. Bucher G. Doumy, B. Erk K. R. Ferguson, T. Gorkhover, P. J. Ho, E. P. Kanter, B. Krassig, J. Krzywinski, A. A. Lutman, A. M. March, D. Moonshiram, D. Ray, L. Young, S. T. Pratt, and S. H. Southworth, Nat. Commun. 7, 11652 (2016). 
Table I. Average Substate-Dependent $4 \mathrm{~d}^{-1}$ Photoelectron Linewidths.

\begin{tabular}{cccccc}
\hline Final State & $\begin{array}{c}\text { Energy }(\mathrm{eV}) \\
\text { Present }\end{array}$ & $\begin{array}{c}\text { Energy (eV) } \\
\text { Ref. 36 }\end{array}$ & $\begin{array}{c}\text { FWHM (eV) } \\
\text { Present }\end{array}$ & $\begin{array}{c}\text { FWHM (eV) } \\
\text { Ref. 22 }\end{array}$ & $\begin{array}{c}\text { FWHM (eV) } \\
\text { Ref. 36 }\end{array}$ \\
\hline \hline $\mathrm{Xe}\left(4 \mathrm{~d}_{5 / 2^{-1}}\right)$ & $67.548(1)^{\mathrm{a}}$ & & $0.109(4)$ & $0.111(3)$ & $0.207(4)$ \\
$\mathrm{Xe}\left(4 \mathrm{~d}_{3 / 2^{-1}}\right)$ & $69.539(5)$ & & $0.102(4)$ & $0.104(3)$ & $0.202(4)$ \\
$\mathrm{XeF}_{2}\left({ }^{2} \Delta 5 / 2\right)$ & $70.169(6)$ & $70.179(6)$ & $0.133(7)$ & & $0.214(19)$ \\
$\mathrm{XeF}_{2}\left({ }^{2} \Pi_{3 / 2}\right)$ & $70.404(6)$ & $70.421(9)$ & $0.127(13)$ & & $0.256(27)$ \\
$\mathrm{XeF}_{2}\left({ }^{2} \Sigma_{1 / 2}\right)$ & $70.579(6)$ & $70.601(13)$ & $0.160(6)$ & & $0.264(26)$ \\
$\mathrm{XeF}_{2}\left({ }^{2} \Delta 3 / 2\right)$ & $72.219(6)$ & $72.248(6)$ & $0.123(5)$ & & $0.223(10)$ \\
$\mathrm{XeF}_{2}\left({ }^{2} \Pi_{1 / 2}\right)$ & $72.538(6)$ & $72.568(6)$ & $0.147(5)$ & & $0.248(8)$ \\
\hline \hline
\end{tabular}

a. The atomic Xe $\left(4 \mathrm{~d}_{5 / 2^{-1}}\right)$ energy was taken from Ref. 5, and was used to calibrate the energy scale. The absolute uncertainty in the calibration energy is $0.01 \mathrm{eV}$. The relative uncertainties for the energies of the other peaks are significantly smaller than the absolute uncertainties. 
Table II. Resonance energies below the $\mathrm{XeF}_{2} 4 \mathrm{~d}^{-1}$ threshold. ${ }^{\mathrm{a}}$

\begin{tabular}{|c|c|c|c|c|c|c|c|}
\hline $\operatorname{Energy}(e V)^{b}$ & Assignment & $\begin{array}{c}\mathrm{n}^{*} \\
\left({ }^{2} \Delta 5 / 2\right) \\
70.169 \\
\mathrm{eV} \\
\end{array}$ & $\begin{array}{c}\mathrm{n}^{*} \\
\left({ }^{2} \Pi_{3 / 2}\right) \\
70.404 \\
\mathrm{eV} \\
\end{array}$ & $\begin{array}{c}\mathrm{n}^{*} \\
\left({ }^{2} \Sigma_{1 / 2}\right) \\
70.579 \\
\mathrm{eV} \\
\end{array}$ & $\begin{array}{c}\mathrm{n}^{*} \\
\left({ }^{2} \Delta 3 / 2\right) \\
72.219 \\
\mathrm{eV} \\
\end{array}$ & $\begin{array}{c}\mathrm{n}^{*} \\
\left({ }^{2} \Pi_{1 / 2}\right) \\
72.538 \\
\mathrm{eV} \\
\end{array}$ & $\begin{array}{c}\text { Energy (eV) } \\
\text { (Ref. 31) }\end{array}$ \\
\hline 61.526 & $\left(4 \mathrm{~d}_{5 / 2^{-1}}\right) \sigma^{*}$ & 1.2547 & 1.2379 & 1.2259 & & & 61.38 \\
\hline 63.455 & $\left(4 \mathrm{~d}_{3 / 2^{-1}}\right) \sigma^{*}$ & & & & 1.2460 & 1.2239 & 63.29 \\
\hline 65.110 & $\mathrm{Xe}\left(4 \mathrm{~d}_{5 / 2^{-1}}\right) 6 \mathrm{p}$ & & & & & & \\
\hline 66.367 & $\mathrm{Xe}\left(4 \mathrm{~d} 5 / 2^{-1}\right) 7 \mathrm{p}$ & & & & & & \\
\hline 67.028 & $\mathrm{Xe}\left(4 \mathrm{~d}_{3 / 2^{-1}}\right) 6 \mathrm{p}$ & & & & & & 66.94 "6s" \\
\hline 67.596 & $\left({ }^{2} \Delta 5 / 2\right) 6 \mathrm{p} \pi$ & 2.2995 & 2.2012 & 2.1357 & & & 67.49 \\
\hline 67.820 & $\left({ }^{2} \Pi_{3 / 2}\right) 6 \mathrm{p} \pi$ & 2.4067 & 2.2946 & 2.2207 & & & 67.73 \\
\hline 67.983 & $\left({ }^{2} \Sigma_{1 / 2}\right) 6 \mathrm{p} \pi$ & 2.4948 & 2.3706 & 2.2893 & & & \\
\hline 68.219 & $\left({ }^{2} \Pi_{3 / 2}\right) 6 \mathrm{p} \sigma$ & 2.6415 & 2.4954 & 2.4011 & & & 68.27 \\
\hline 68.351 & $\left({ }^{2} \Sigma_{1 / 2}\right) 6 \mathrm{p} \sigma$ & 2.7357 & 2.5743 & 2.4712 & & & \\
\hline 68.961 & $\left({ }^{2} \Delta_{5 / 2}\right) 7 \mathrm{p} \pi$ & 3.3560 & 3.1579 & 2.8998 & & & 69.00 "6s" \\
\hline 69.189 & $\left({ }^{2} \Pi_{3 / 2}\right) 7 \mathrm{p} \pi$ & 3.7260 & 3.3464 & 3.1286 & & & \\
\hline 69.433 & $\left({ }^{2} \Sigma_{1 / 2}\right) 7 \mathrm{p} \pi$ & 4.2995 & 3.7433 & 3.4456 & & & \\
\hline 69.617 & $\left({ }^{2} \Delta 3 / 2\right) 6 \mathrm{p} \pi$ & & & & 2.2867 & 2.1582 & 69.53 \\
\hline 69.942 & $\left({ }^{2} \Pi_{1 / 2}\right) 6 \mathrm{p} \pi$ & & & & 2.4444 & 2.2893 & 60.84 \\
\hline 70.029 & $\left({ }^{2} \Delta 3 / 2\right) 6 \mathrm{p} \sigma$ & & & & 2.4925 & 2.3287 & 70.20 \\
\hline 70.338 & $\left({ }^{2} \Pi_{1 / 2}\right) 6 \mathrm{p} \sigma$ & & & & 2.6895 & 2.4868 & \\
\hline 70.994 & $\left({ }^{2} \Delta_{3 / 2}\right) 7 \mathrm{p} \pi$ & & & & 3.3327 & 2.9685 & 70.89 \\
\hline 71.328 & $\left({ }^{2} \Pi_{1 / 2}\right) 7 \mathrm{p} \pi$ & & & & 3.9077 & 3.3533 & \\
\hline 71.472 & $\left({ }^{2} \Delta_{3 / 2}\right) 7 \mathrm{p} \sigma$ & & & & 4.2678 & 3.5736 & 71.47 \\
\hline
\end{tabular}


a. The $n^{*}$ values were calculated by using Equation 5, the ionization energies at the top of each column, and the Rydberg constant for $\mathrm{XeF}_{2}(13.6056 \mathrm{eV})$.

b. The relative uncertainty in the line positions is $0.005 \mathrm{eV}$, and the absolute uncertainty from the calibration line is $0.01 \mathrm{eV} .^{5}$ 
Table III. Valence Shake-Up Processes.

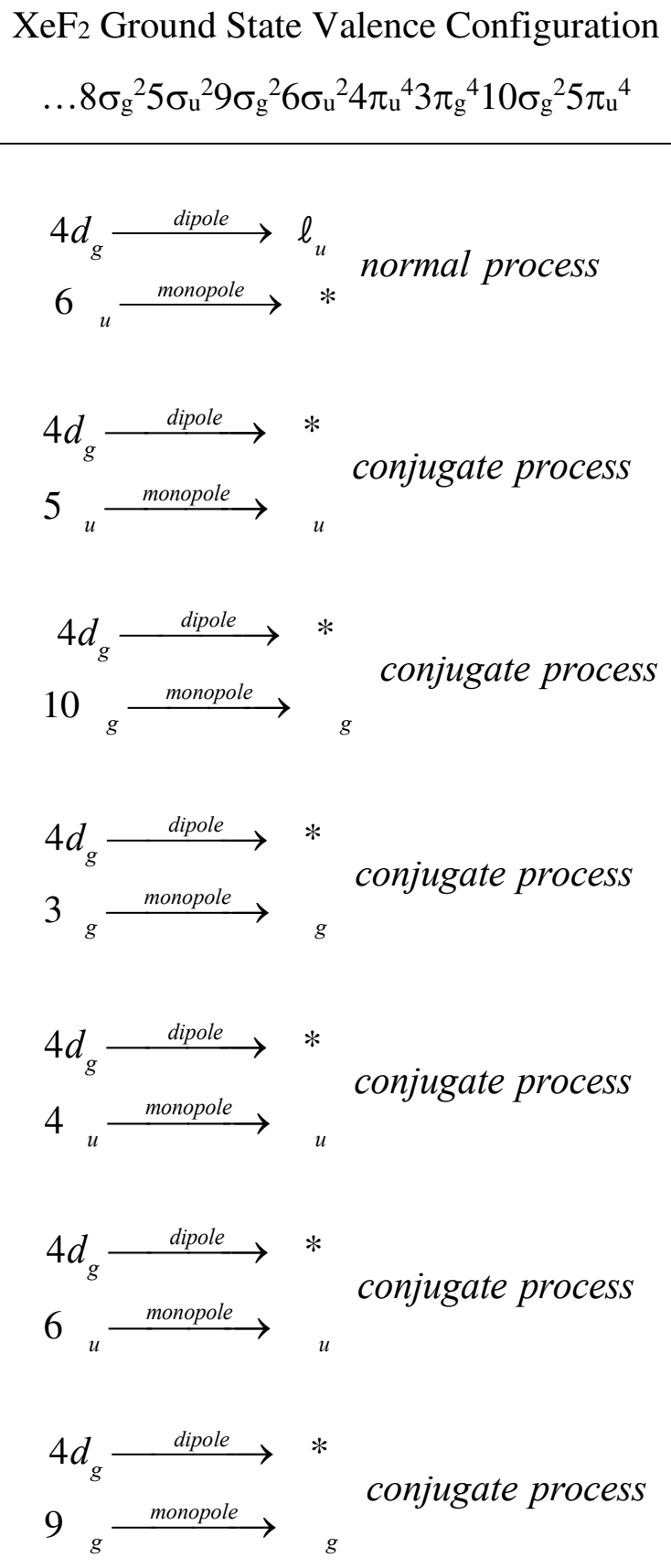


Table IV. Auger-Electron Energies and Tentative Assignments.

\begin{tabular}{|c|c|c|}
\hline $\begin{array}{c}\text { Peak Number } \\
\left.\text { (Partner }^{\text {Number) }}\right)^{\mathrm{a}}\end{array}$ & $\begin{array}{c}\text { Kinetic Energy } \\
(\mathrm{eV})\end{array}$ & $\begin{array}{c}\text { Initial Xe } \\
\text { Spin-Orbit State }\end{array}$ \\
\hline $1(5)$ & 39.64 & $4 d_{3 / 2}$ \\
\hline 2 & 39.34 & $4 d_{3 / 2}$ \\
\hline $3(6)$ & 38.84 & $4 d_{3 / 2}$ \\
\hline 4 & 37.83 & $4 d_{3 / 2}$ \\
\hline $5(1)$ & 37.58 & $4 d_{5 / 2}$ \\
\hline $6(3)$ & 36.87 & $4 d_{5 / 2}$ \\
\hline 7 & 36.24 & \\
\hline 8 & 35.12 & \\
\hline 9 & 34.34 & \\
\hline $10(12)$ & 33.24 & $4 d_{3 / 2}$ \\
\hline $11(13)$ & 31.89 & $4 d_{3 / 2}$ \\
\hline $12(10)$ & 31.25 & $4 d_{5 / 2}$ \\
\hline $13(11)$ & 29.88 & $4 d_{5 / 2}$ \\
\hline $14(15)$ & 26.64 & $4 d_{3 / 2}$ \\
\hline $15(14)$ & 24.27 & $4 d_{5 / 2}$ \\
\hline
\end{tabular}

a. The numbers in parentheses indicate the paired level decaying to same final state. 


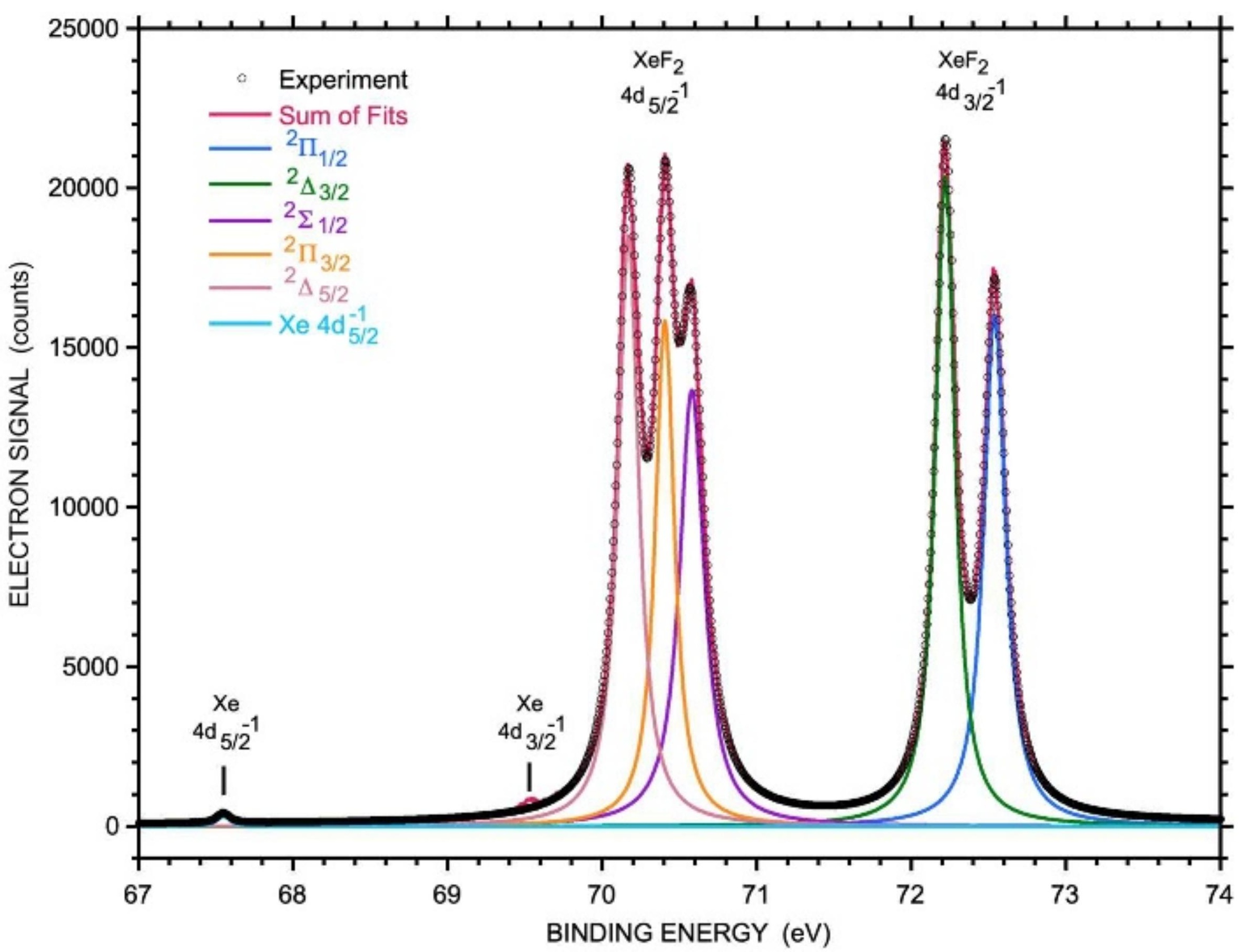



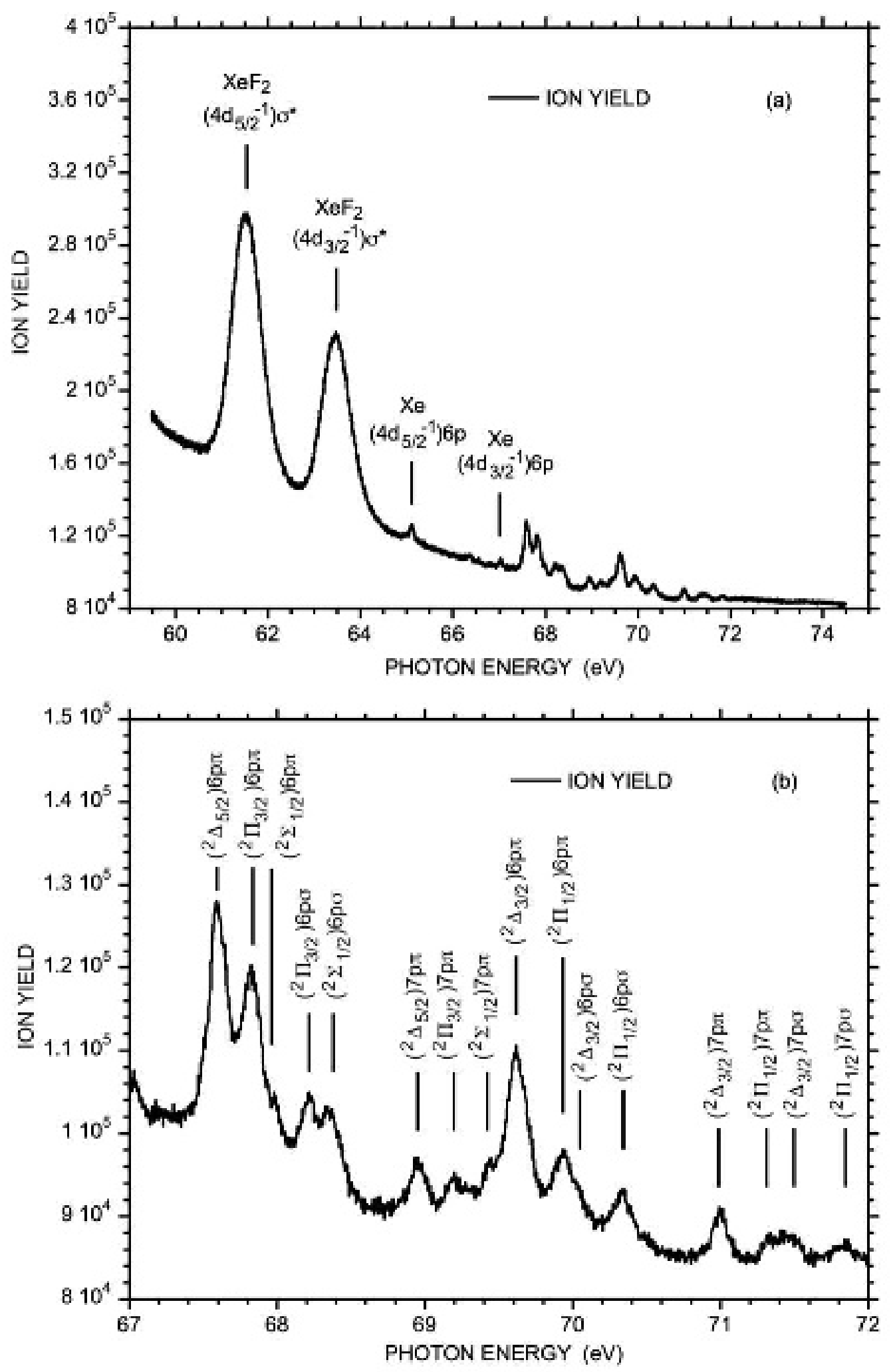

Figure 2 

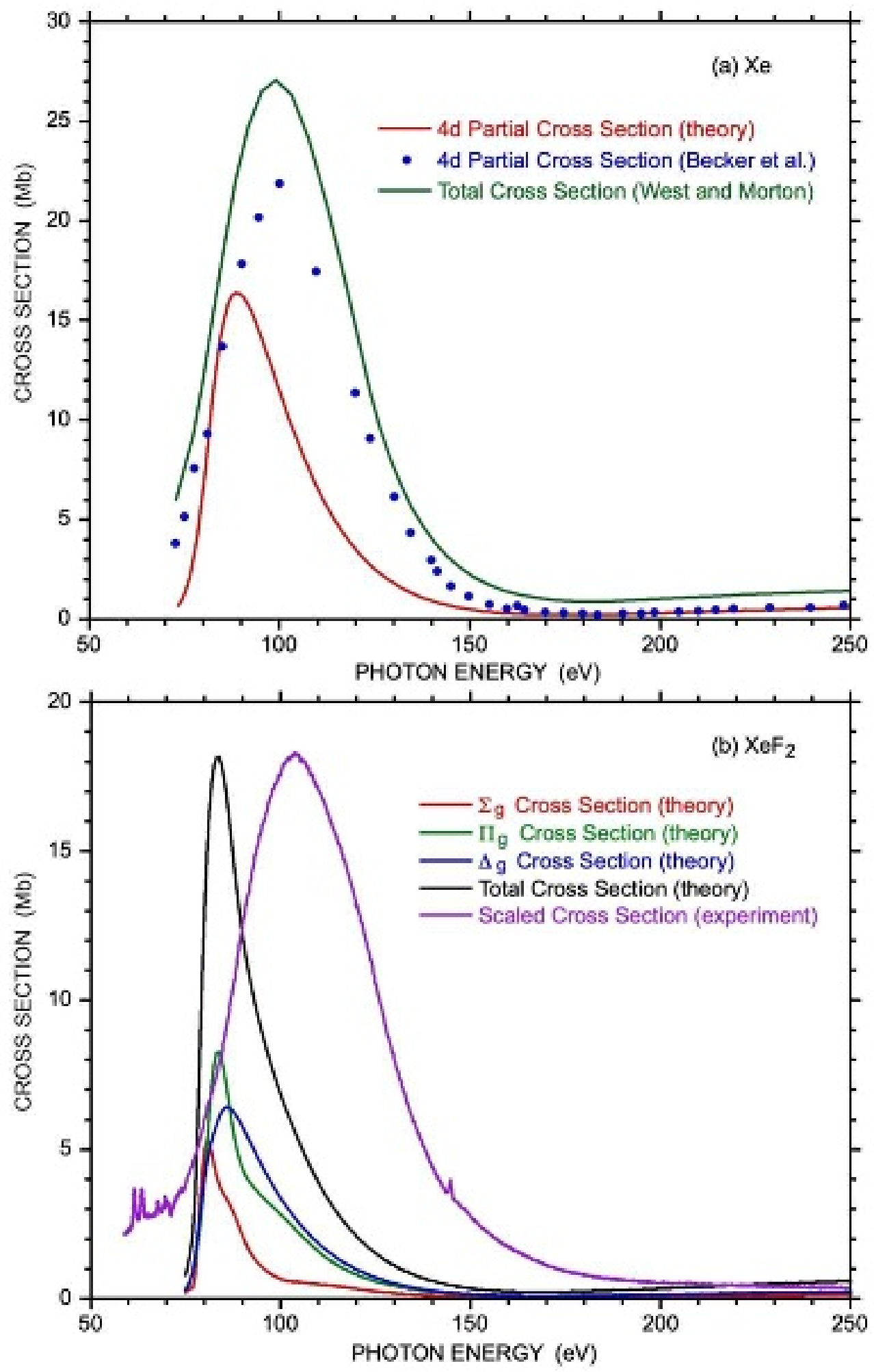

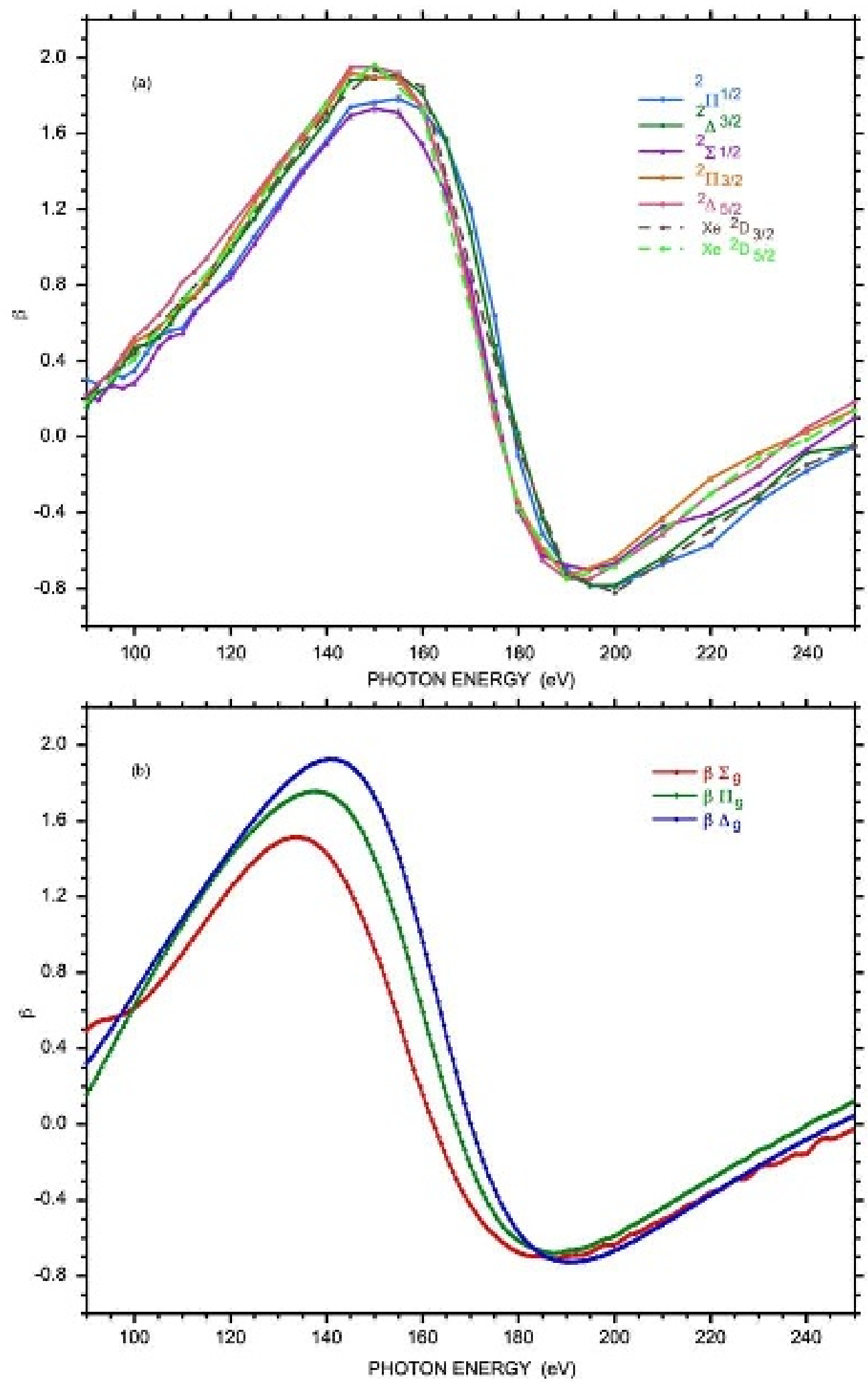

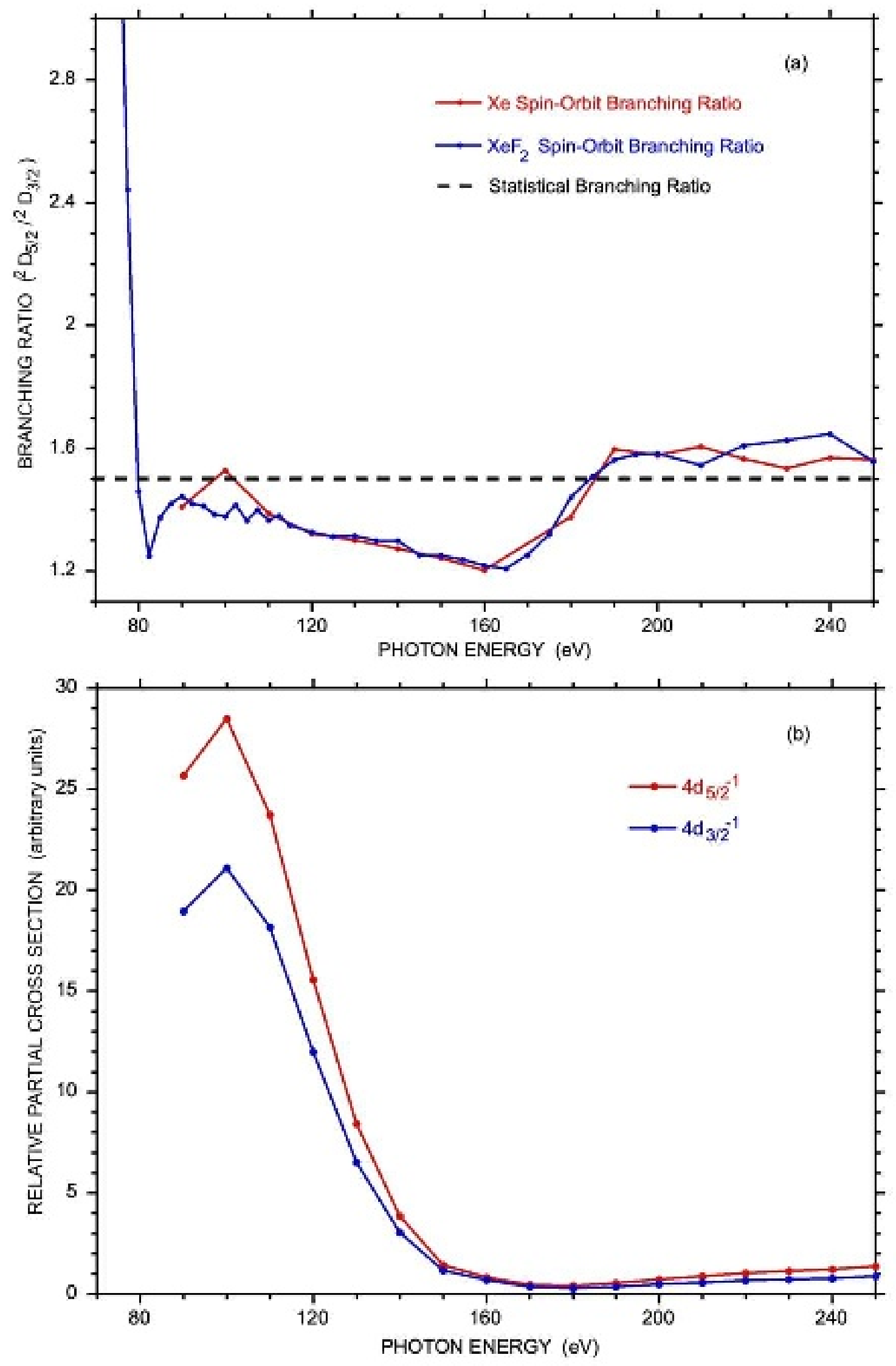


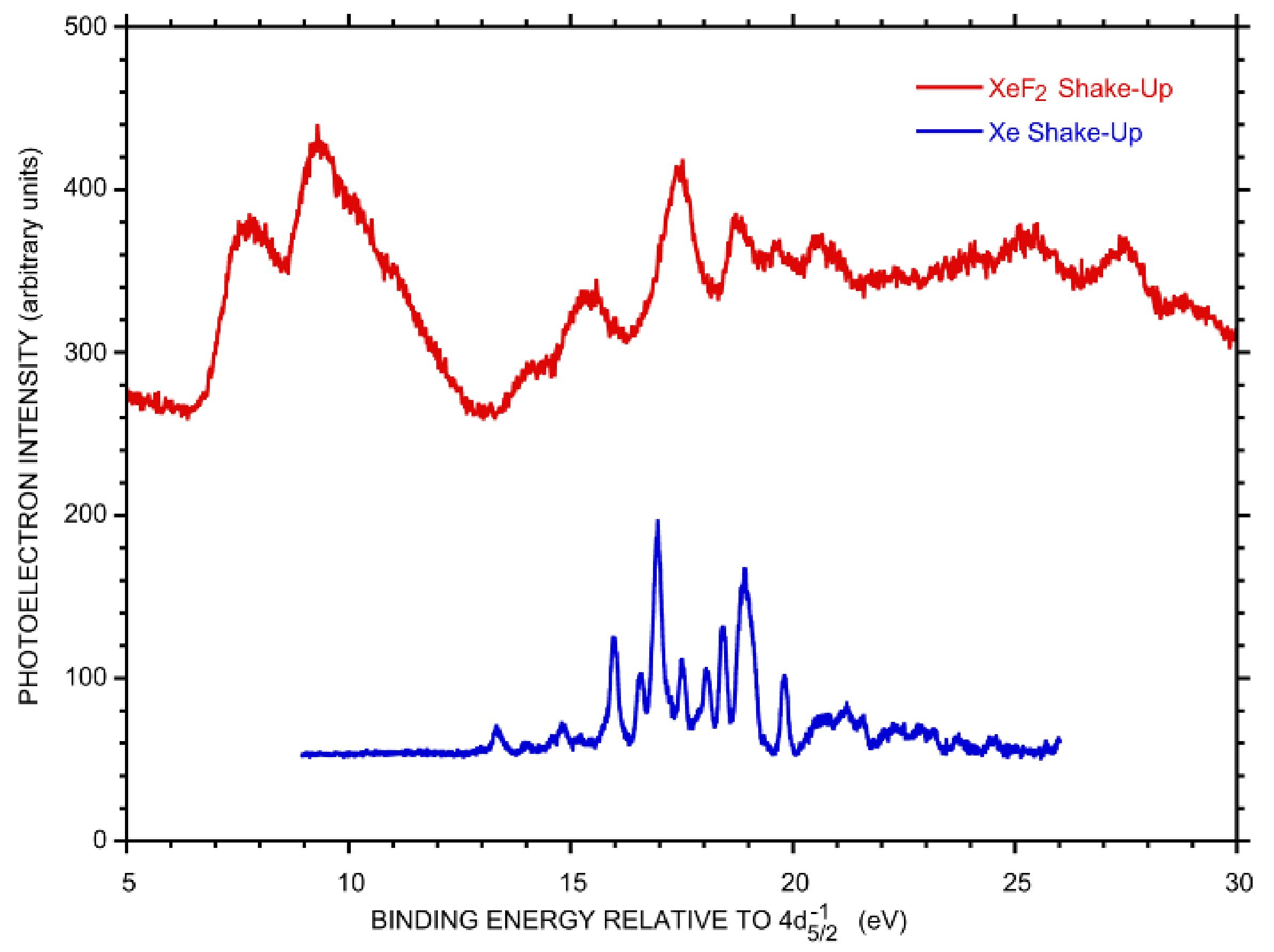



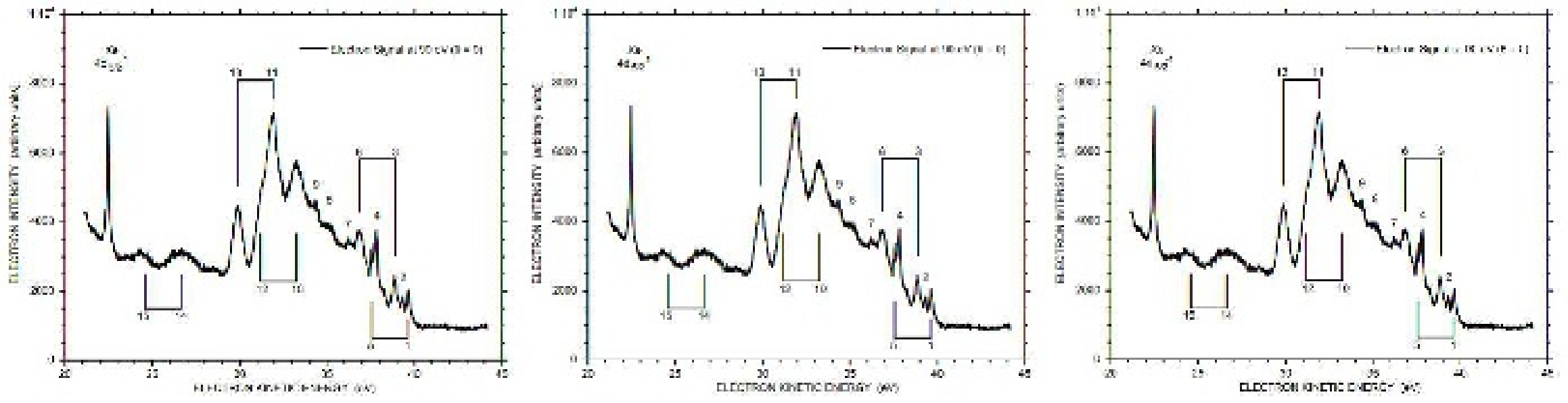


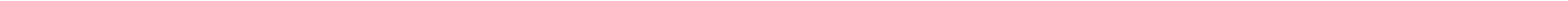




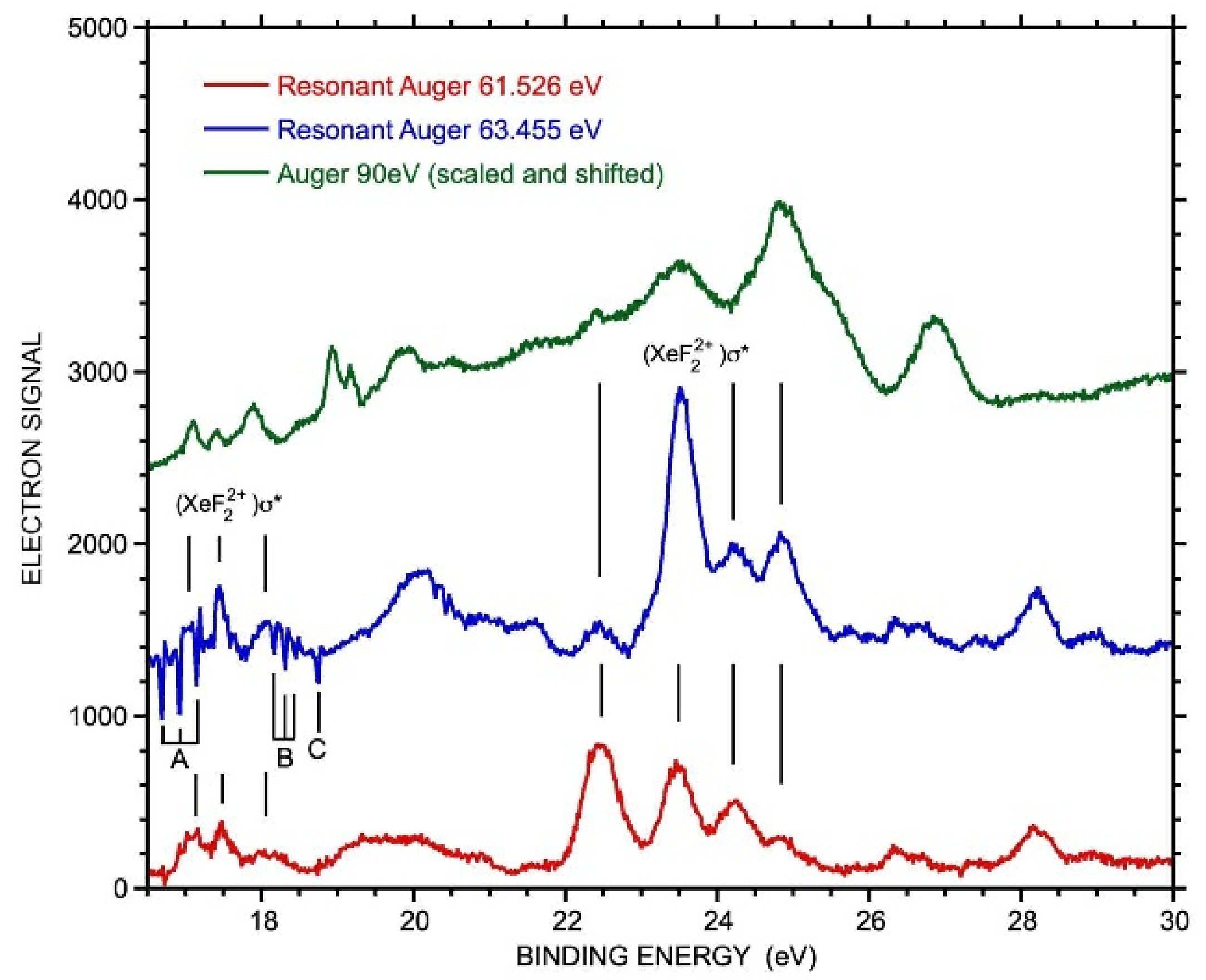

